\title{
Kinetic modeling and parametric imaging with dynamic PET for oncological applications: general considerations, current clinical applications, and future perspectives
}

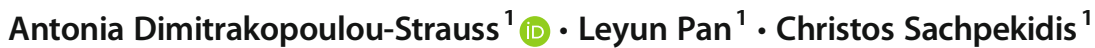

Received: 17 January 2020 / Accepted: 27 April 2020 / Published online: 19 May 2020

(C) The Author(s) 2020

\begin{abstract}
Dynamic PET (dPET) studies have been used until now primarily within research purposes. Although it is generally accepted that the information provided by dPET is superior to that of conventional static PET acquisitions acquired usually 60 min post injection of the radiotracer, the duration of dynamic protocols, the limited axial field of view (FOV) of current generation clinical PET systems covering a relatively small axial extent of the human body for a dynamic measurement, and the complexity of data evaluation have hampered its implementation into clinical routine. However, the development of new-generation PET/CT scanners with an extended FOV as well as of more sophisticated evaluation software packages that offer better segmentation algorithms, automatic retrieval of the arterial input function, and automatic calculation of parametric imaging, in combination with dedicated shorter dynamic protocols, will facilitate the wider use of dPET. This is expected to aid in oncological diagnostics and therapy assessment. The aim of this review is to present some general considerations about dPET analysis in oncology by means of kinetic modeling, based on compartmental and noncompartmental approaches, and parametric imaging. Moreover, the current clinical applications and future perspectives of the modality are outlined.
\end{abstract}

Keywords Dynamic PET $\cdot$ Oncology $\cdot$ Kinetic modeling $\cdot$ Parametric imaging $\cdot$ Feature extraction

\section{Introduction}

Positron emission tomography (PET) is a tomographic, quantitative, imaging method providing information on biochemical processes in vivo. PET was developed in the late 1970s, and its first applications were brain studies using the radiotracer 2-deoxy-2- $\left({ }^{18} \mathrm{~F}\right)$ fluoro-D-glucose $\left({ }^{18} \mathrm{~F}\right.$-FDG) [1]. In the late 1980s, PET was introduced into oncological studies based mostly on ${ }^{18}$ F-FDG.

The first PET measurements were based on dynamic imaging protocols for the evaluation of tracer pharmacokinetics particularly in the brain. These initial dynamic PET (dPET)

This article is part of the Topical Collection on Advanced Image Analyses (Radiomics and Artificial Intelligence)

Antonia Dimitrakopoulou-Strauss

a.dimitrakopoulou-strauss@dkfz.de

1 Clinical Cooperation Unit Nuclear Medicine, German Cancer Research Center, Im Neuenheimer Feld 280,

69120 Heidelberg, Germany acquisitions were confined to a single-bed position, thus limiting their wide clinical application especially in the field of oncology where metastatic disease evaluation calls for wholebody (or at least multibed) PET imaging protocols. Quantitative imaging evaluation was performed by the calculation of time activity curves (TACs), areas under the curve (AUCs), standardized uptake values (SUVs) as well as on more complex kinetic modeling approaches depending on the tracer used. In the case of ${ }^{18} \mathrm{~F}-\mathrm{FDG}$, the generally accepted method for accurate analysis of the tracer's kinetics is a compartment model originally proposed by Sokoloff et al., developed to measure 2-deoxy-D- $\left({ }^{14} \mathrm{C}\right)$ glucose in the rat brain tissue by autoradiography [2]. A few years later, Phelps et al. proposed a modified 3-tissue compartment model for the calculation of the transport rates of ${ }^{18} \mathrm{~F}$-FDG, namely $K_{1}$ and $k_{2}$ which represent the transport rate of ${ }^{18} \mathrm{~F}-\mathrm{FDG}$ into the tissue and reverse, as well as $k_{3}$ and $k_{4}$ that reflect the phosphorylation and dephosphorylation rate of the tracer. This modified model is actually an extension of the one originally proposed by Sokoloff et al. The difference between them lies in the calculation of $k_{4}$ in the model by Phelps et al., which is considered negligible in the Sokoloff model [3]. Since then, 
several assumptions and modifications have been made to the original mathematical formulations and the model has been successfully applied for the assessment of ${ }^{18} \mathrm{~F}$-FDG dPET studies in tumors [4].

Approximately 20 years ago, the conventional PET scanners were replaced by hybrid PET/CT systems, which combine diagnostic information of two modalities. Moreover, the recent development of the novel PET/MRI technology represents a very promising candidate in hybrid imaging, although its role in the clinical setting remains to be determined. The advent of hybrid imaging technology provides superior information due to the combination of structural and functional tomographic imaging modalities. This new era facilitated the performance of whole-body PET/CT scans in a relatively short time- - less than 30 min-and with high image quality, leading to the widespread use of the modality in clinical practice. In particular in oncology, PET/CT is considered nowadays the standard imaging technique for diagnosis, staging, and monitoring of several different tumor types.

The vast majority of PET/CT studies are based on acquisition and visual evaluation of static, late, whole-body images usually 60 min post-injection (p.i.) of the radiotracer, occasionally employing semi-quantitative analysis based on calculation of SUV values. SUV represents tissue activity within a region of interest (ROI) corrected for injected activity and body weight, and is the most widely used method for quantification of PET data, since its calculation requires only static imaging, after the tracer is assumed to have reached its equilibrium. SUV calculation is nowadays available in commercial imaging software packages. SUV is, however, dependent on many different factors such as the time interval between injection and scanning as well as different image acquisition settings characteristics (scanner, scatter and attenuation correction, reconstruction algorithm, frame duration), rendering the comparison of SUV values acquired in different centers problematic, when even slight differences in the acquisition procedure are present [5]. Moreover, tracer uptake $60 \mathrm{~min}$ p.i. is the result of a dynamic process. One important aspect of PET is the possibility of performing accurate, noninvasive quantitative measurements of tracer concentration in patients, which requires the performance of a dPET study usually with a duration of $60 \mathrm{~min}$, in addition to the regular static PET/CT scan.

Although it is generally accepted that the information provided by $\mathrm{dPET}$ is superior to that of conventional static PET acquisitions, several issues have hampered its implementation into clinical routine. Apart from the longer duration of dynamic protocols, some major limitations include the limited axial field of view (FOV) of the current generation clinical PET systems and the fact that the majority of dPET protocols are still confined to a single-bed axial FOV. These issues are beginning to be addressed with the development and introduction of clinical PET systems with gradually longer-more than $1 \mathrm{~m}$-axial FOVs, better electronics, and resolution
[6-10]. Moreover, the introduction of clinically feasible dynamic whole-body PET imaging protocols in current generation limited axial FOV PET systems equipped with direct 4D reconstruction schemes and generalized nonlinear graphical analysis methods has rendered graphical analysis (Patlak) whole-body dPET and parametric imaging possible [11-15].

These developments will improve the statistical quality of PET images and allow whole-body scanning in shorter time, and may, subsequently, lead to a potential renaissance of dPET studies with the perspective of even performing whole-body pharmacokinetic studies. dPET scanning can provide reliable assessments of dedicated metabolic steps of metabolic active tracers, such as ${ }^{18} \mathrm{~F}-\mathrm{FDG}$, as well as newer receptor-binding agents such as radiolabeled prostate-specific membrane antigen (PSMA) or DOTATOC ligands with potential applications in the rapidly evolving field of radiothera(g)nostics. Moreover, the widespread application of dPET would be of great importance in the evaluation of new (radio)pharmaceutical agents.

The aim of this review is to present the general principles of dPET data analysis in oncology by means of kinetic modeling, based on compartmental and noncompartmental approaches, as well as by parametric imaging. Moreover, the current clinical applications and future perspectives of dPET will be outlined.

\section{dPET studies: general considerations}

\section{Volume of interest-based analysis}

Workflow, protocol dPET studies require a dynamic acquisition for a certain time depending on the pharmaceutical and the radionuclide used. The target area for APET should be defined carefully, usually including the anatomical region with the known or suspicious tumor lesions. The advent of the newgeneration PET scanners with extended FOV will simplify the choice of a target area by allowing dynamic acquisition of almost the whole body. For ${ }^{18} \mathrm{~F}$-FDG, the most common used PET tracer in oncology, 60 min of dynamic acquisition are required. Similarly, 60-min dynamic acquisitions are also applied for receptor-binding tracers, such as DOTATOC and PSMA radioligands $[16,17]$. For transport tracers, like ${ }^{11} \mathrm{C}$ labeled amino acids (e.g., C-11-methionine or C-11-choline)) a shorter acquisition protocol, e.g., for $20-30 \mathrm{~min}$, is usually applied. The dynamic imaging is acquired in a list mode, and then, frames are defined by the users (Fig. 1). The frame duration should be short for the first frames and increase during the progress of the dPET acquisition (e.g., $10 \mathrm{~s}$, followed by $30 \mathrm{~s}$, $60 \mathrm{~s}, 120 \mathrm{~s}$, and $300 \mathrm{~s})$.

Definition of tumor VOls The evaluation of the reconstructed PET images is based on visual analysis of PET and fused PET/ CT or PET/MRI images. Tumor volume of interest (VOI) is then placed over areas with increased tracer uptake as compared with the surrounding tissue, after correlation with the 
Fig. 1 Transversal (left) and coronal (right) images of a dPET series of the thorax following i.v.

${ }^{18} \mathrm{~F}$-FDG injection at $1,12,16$,

30 , and $60 \mathrm{~min}$ p.i. in a patient with metastases (mediastinal, lung, and liver) from melanoma. Visualization of the vessels in the early images and gradually increasing ${ }^{18} \mathrm{~F}$-FDG uptake in the metastases in the following frames

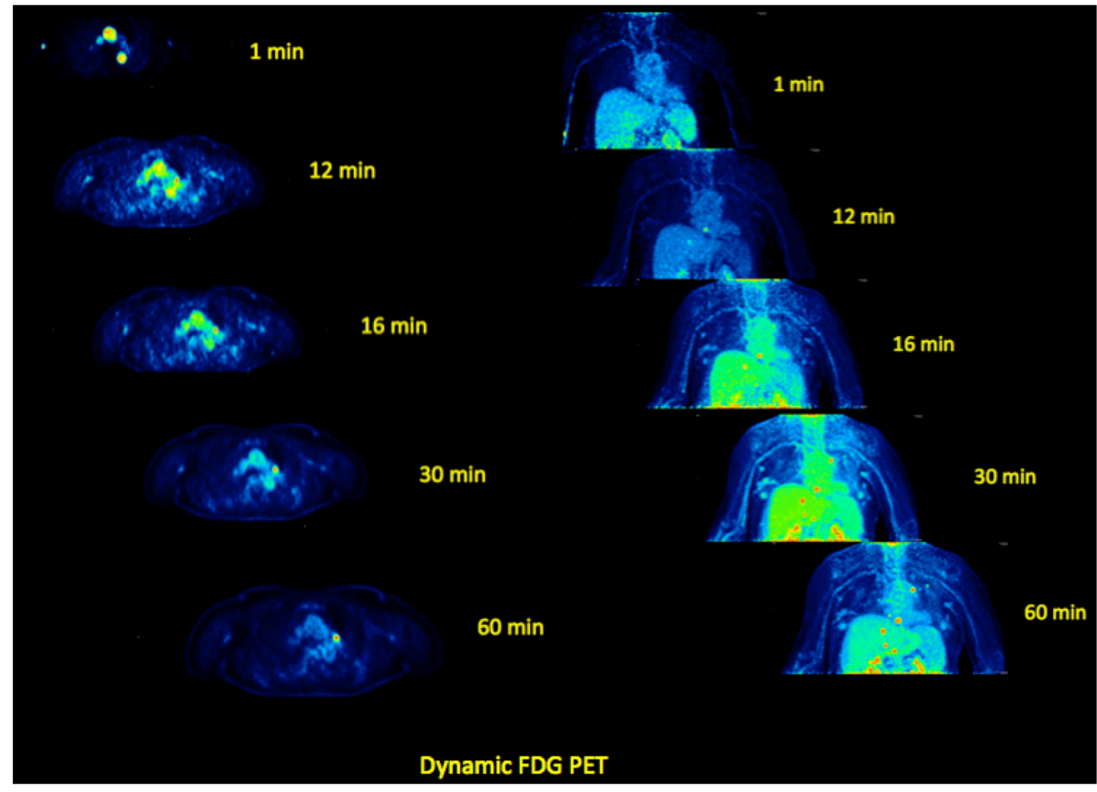

CT/MRI images and using dedicated algorithms, usually an isocontour. VOIs drawn over reference tissue without pathologically increased tracer uptake are also recommended for comparison.

Input function: general considerations and problems The accurate assessment of the arterial input function is a topic broadly investigated in the literature. Manual blood sampling at different time points p.i. or continuous automatic blood sampling during the whole dynamic acquisition have been used in limited number of studies [18]. Phelps et al. first reported on a good agreement between arterial blood sampling and arterialized venous sampling by using hand heating to $44^{\circ} \mathrm{C}$ [3]. Although, arterial blood sampling is considered the gold standard for input function measurements, it is an invasive procedure and cannot be recommended for routine clinical purposes. Furthermore, delay in terms of different appearance time of the radioactivity after a bolus injection and dispersion affects the time activity curve used for input. In particular, when using blood sampling, the measured blood curve is smeared due to inhomogeneous velocity in the vessels and the catheter and due to sticking of radiotracer in the catheter tubes used. Therefore, dispersion correction is recommended in particular for tracers with very fast kinetics, like perfusion studies [19, 20].

Double supply of organs like the liver causes special problems in the estimation of the input function. In particular, the liver receives blood supply from both the portal vein and the hepatic artery with the TAC of the portal vein being delayed and dispersed as compared with the hepatic artery. Keiding reported on methods of estimating the dual-input TAC without portal vein measurements in particular for measurements of the regional hepatic blood perfusion [21].
Noninvasive devices have been introduced for the determination of the arterial input function. Recently, Turgeon et al. introduced a detector of scintillating fiber coupled to transmission fiber-optic cables, which are connected to photomultiplier tubes as a device that can be wrapped around the wrist of the patient studied with PET [22]. The results are promising and suggest that scintillating fibers may be used for the noninvasive measurement of the arterial input function.

However, the most attractive, easy, und user-friendly approach for input function assessment, which also possesses a good accuracy, is its image-derived calculation during the PET scan [23]. Input VOIs can be acquired using the reconstructed images of the first frame(s) drawn over a large arterial vessel like the descending aorta (Fig. 2). An input VOI should include the hottest pixels of the vessel in several sequential slices. Visual inspection should then be used for a first qualitative assessment of the respective TACs: an input VOI should demonstrate a clear activity peak and not be very noisy. In case of much noise, for example due to the use of a smaller vessel, the curve data should be fitted by using a sum of up to three decaying exponentials to reduce noise [24, 25]. For smaller vessels (diameter $<8 \mathrm{~mm}$ ), like the femoral artery, a partial volume correction may be done using the CT data and on the basis of phantom measurements of the recovery function. In recent years, novel approaches for calculation of image-derived arterial input function using integrated PET/ $\mathrm{CT}$ and PET/MRI images have been developed with very promising results $[26,27]$.

Another approach is the use of a standardized, populationbased input function, which is a normalized average of measured arterial blood samples from several subjects [28, 29]. Furthermore, hybrid statistical approaches have been published for the noninvasive assessment of the input function. 
Fig. 2 Patient of Fig. 1. Fused ${ }^{18} \mathrm{~F}$-FDG PET/CT late image (upper right) approximately 80 min p.i. demonstrating an ${ }^{18} \mathrm{~F}$ FDG avid mediastinal lymph node metastasis. Input and tumor VOIs in the descending aorta and the mediastinal lesion for evaluation of the kinetic data of the tracer (left upper and middle row, respectively). Time activity curves of both VOIs and results of kinetic analysis based on a 3tissue compartment model (lower row)
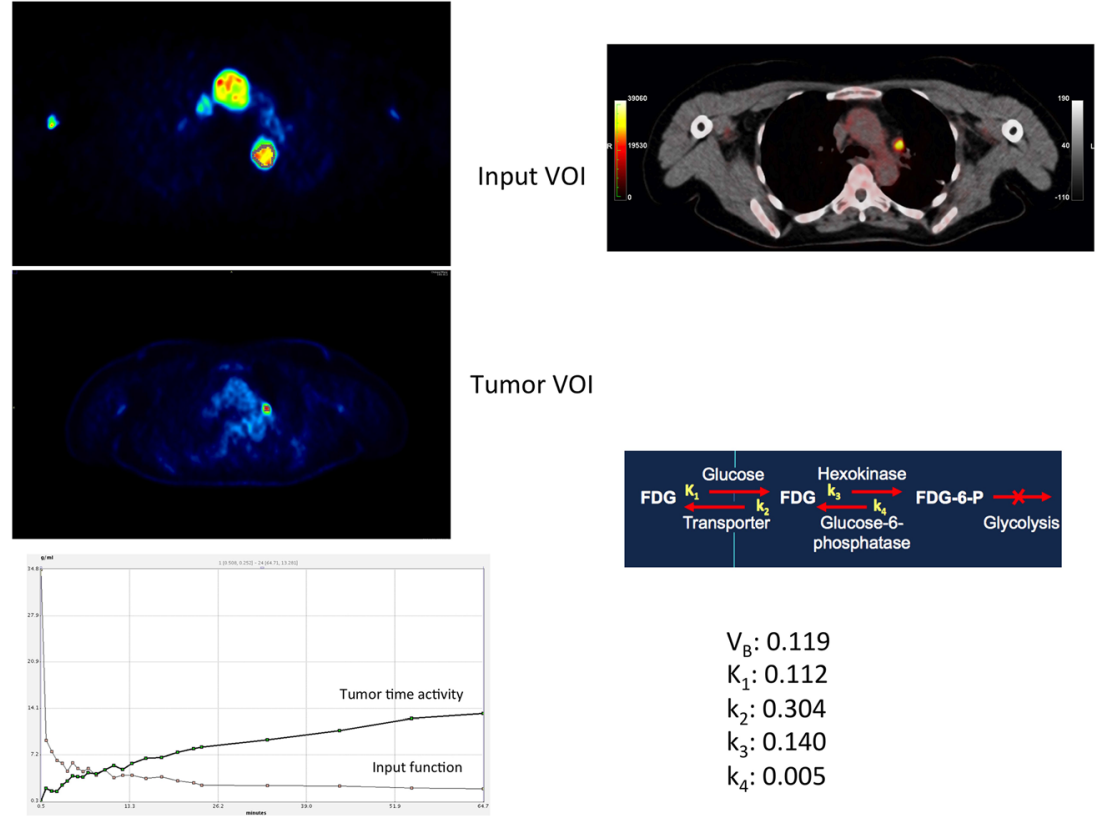

$V_{B}: 0.119$

$\mathrm{K}_{1}: 0.112$

$k_{2}: 0.304$

$\mathrm{k}_{3}: 0.140$

$k_{4}: 0.005$
O'Sullivan et al. proposed a penalty formulation in which the information derived from priori studies is combined in a Bayesian manner with information contained in the sampled image data and recommend this method for clinical use [30].

Lumped constant The difference between ${ }^{18} \mathrm{~F}$-FDG and glucose in terms of transport, phosphorylation, and distribution volume is taken into consideration by using a correction term, the so-called lumped constant $\left(\mathrm{LC}_{\mathrm{FDG}}\right)$. The accuracy of the metabolic rate of ${ }^{18} \mathrm{~F}$-FDG depends on the knowledge of the exact value of LC. Sokoloff et al. measured the LC for ${ }^{14} \mathrm{C}$ deoxyglucose in the rat brain and reported a value of 0.46 [1]. Reivich et al. found a value of 0.52 in human brain [31]. Hasselbalch et al. reported on a mean value of $0.81 \pm 00.15$ in normal healthy volunteers [32]. However, for simplification reasons, LC is considered to be equal to one.

\section{Compartment modeling}

The idea of compartment modeling of PET data has its roots in pharmacology and biochemistry. By providing information on the transport or other metabolic steps of the applied radiopharmaceutical, compartment modeling aims to estimate biologically relevant parameters. However, in vivo dynamic imaging is more complex than in vitro studies partly due to the fact that the uptake of a radiopharmaceutical depends on the vessel density of a tissue. The most commonly used compartment models in PET are the 2-tissue and 3-tissue compartment models (Fig. 3). A simplification of these models consists of the summation of the interstitial and the cellular space. A 2tissue compartment model is suitable for radiopharmaceuticals, which are purely transport markers, like labeled water or amino acids that do not undergo further metabolic steps. A 3-tissue compartment model is appropriate for radiopharmaceuticals, which are transported and then undergo one metabolic step, like ${ }^{18} \mathrm{~F}$-FDG. This model involves the plasma compartment $\mathrm{C}_{\mathrm{p}}$, the exchanging compartment $\mathrm{C}_{1}$ in which the tracer is considered free and nonspecifically bound tracer in tissue (nondisplaceable compartment), and the compartment $\mathrm{C}_{2}$ involving the specifically bound tracer. The arterial plasma $C_{p}$ exchanges with the first tissue compartment $C_{1}$, which in turn exchanges with the second tissue compartment
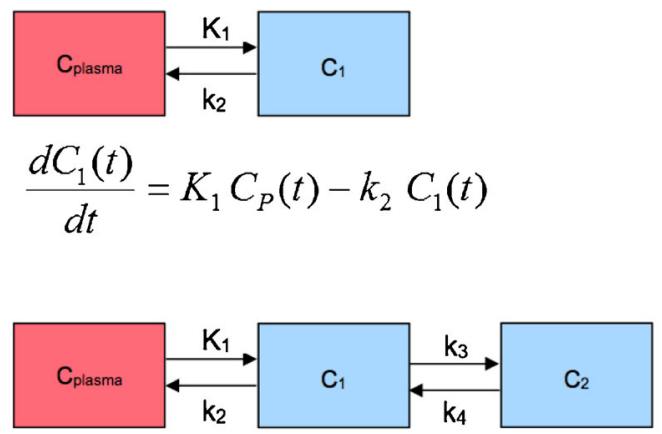

$$
\begin{array}{ccc}
\frac{d C_{1}(t)}{d t}= & K_{1} C_{P}(t)-\left(k_{2}+k_{3}\right) C_{1}(t)+k_{4} C_{2}(t) \\
\frac{d C_{2}(t)}{d t}= & k_{3} C_{1}(t)-k_{4} C_{2}(t)
\end{array}
$$

Fig. 3 Schematic presentation of the 2-and 3-compartment model with one input function. $C_{\text {plasma }}$ is the tracer concentration in arterial blood. In the 2-tissue compartment, all tracer is transported in compartment $C_{1}$. For the 3-tissue compartment, two tracer forms in tissue are considered as $C_{1}$ and $C_{2}$. Compartment $C_{1}$ represents the free and nondisplaceable part of the tracer into the tissue, and compartment $C_{2}$ represents the specific bound part of the tracer (in case of ${ }^{18} \mathrm{~F}$-FDG the phosphorylated tracer) 
$\mathrm{C}_{2}$. The application of the 3-tissue compartment model leads to the extraction of the kinetic parameters $K_{1}$ and $k_{2}$, which are the uptake and clearance rate constants (i.e., between $C_{p}$ and $C_{1}$ ), as well as of the parameters $k_{3}$ and $k_{4}$, which describe the exchange between the tissue compartments $C_{1}$ and $C_{2}$ (Fig. 3) (http://doc.pmod.com/pkin/pkin.html). The unit for these rate constants is $1 / \mathrm{min}$.

In the case of ${ }^{18} \mathrm{~F}-\mathrm{FDG}$, it is known that the tracer is transported by different glucose transporters, phosphorylated by different hexokinases and then trapped intracellularly without undergoing further metabolic steps [33, 34]. According to the 3-tissue compartment model, four transport rates describe the exchange of the radiopharmaceutical between the blood and the tissue compartments. Appropriate algorithms, like Marquardt-Levenberg, allow an estimation of the fractional blood volume, also known as vessel density $\left(V_{B}\right)$, as well as the above-described transport rates of ${ }^{18}$ F-FDG $K_{1}, k_{2}, k_{3}$, and $k_{4}: K_{1}$ is related to the influx of the tracer from the blood compartment to the tissue compartment, $k_{2}$ is related to the efflux, $k_{3}$ reflects the phosphorylation rate, and $k_{4}$ reflects the dephosphorylation rate. $V_{B}$ and $K_{1}$ are related and are typically higher than the phosphorylation rate $k_{3}$. One should keep in mind that the rates assessed by the compartment modeling are a compromise between a pure mathematical solution and biological and practical limitations of the method. It is recommended to use an upper limit of 1 for each transport rate, while $V_{B}$ values should exceed 0 , even if from a mathematical point of view different values may be occasionally calculated. This model is different than the one proposed by Sokoloff et al., which does not take into consideration $k_{4}$ and $V_{B}$. The lack of $k_{4}$ and $V_{B}$ leads to different $K_{1}$ and $k_{3}$ values, since $K_{1}$ is dependent on $V_{B}$, and $k_{3}$ dependent on $k_{4}$. Moreover, the dephosphorylation rate $\left(k_{4}\right)$ of ${ }^{18} \mathrm{~F}$-FDG may be low, but is not negligible. Details about the implementation of different compartment models and the software requirements for clinical use are described by Burger and Buck [35] (https://www. pmod.com/web/?portfolio=11-modeling-pkin). Of note is that this model may be confounded in kidney evaluations by urinary excretion.

A limitation of compartment modeling is that the assessment of the transport rates is operator-dependent and should be performed only by experienced users. The reason is that these models use an iterative fitting (IF) to calculate the least squares between measured and model data, which may lead to overfitting problems and lack of reproducibility. Noise in the TACs and, in particular, inappropriate input TACs have an impact on the assessed rates. A solution to overcome these problems has been published by a group, based on machine learning approaches and oncological reference databases with a training set of modeling data. In specific, we have introduced a machine learning (ML)-based kinetic modeling (KM) method, that utilizes a historical reference database to build a kinetic model directly dealing with noisy data but not trying to smooth image noise. Based on the plethora of data in the reference database, this approach can automatically adjust the models using a multithread grid parameter searching technique. Moreover, in an attempt to combine the advantages of ML and IF modeling methods, a candidate competition concept has been developed, which can find a balance between fitting to history data and to unseen target curve. The MLbased method provides a robust and reproducible solution that is user-independent for VOI-based and pixelwise quantitative analysis of PET data [36].

Other, more complicated models with two input functions and five compartments have also been proposed for metabolically active tracers, like ${ }^{11} \mathrm{C}$-thymidine, ${ }^{11} \mathrm{C}$-acetate, or ${ }^{18} \mathrm{~F}$ fluorodeoxythymidine (FLT) (Fig. 4) [37]. These tracers produce labeled circulating metabolites during the dynamic data acquisition due to biochemical breakdown or conjugation shortly after injection. The measurement of these circulating metabolites and the knowledge of their biological behavior in terms of the metabolic processes in which they may be involved is a challenge. Therefore, model input functions of not only the injected radiotracer but also its labeled metabolites should be used for more accurate quantitative assessment. Such a model example is provided for ${ }^{18} \mathrm{~F}$-FLT and its circulating metabolite ${ }^{18}$ F-FLT-glucuronide by Muzi et al. [38]. However, these approaches are very complicated and, thus, not recommended for clinical use. In an attempt to address this issue, more simplified models have been proposed for the characterization of these agents. For example, Shields et al. assessed a 3-tissue compartment model for ${ }^{18} \mathrm{~F}$-FLT with an image-derived input function of the descending aorta and metabolite correction measured in a single sample obtained at $60 \mathrm{~min}$ p.i. They reported that at $60 \mathrm{~min}$ p.i., $74 \%$ of the blood

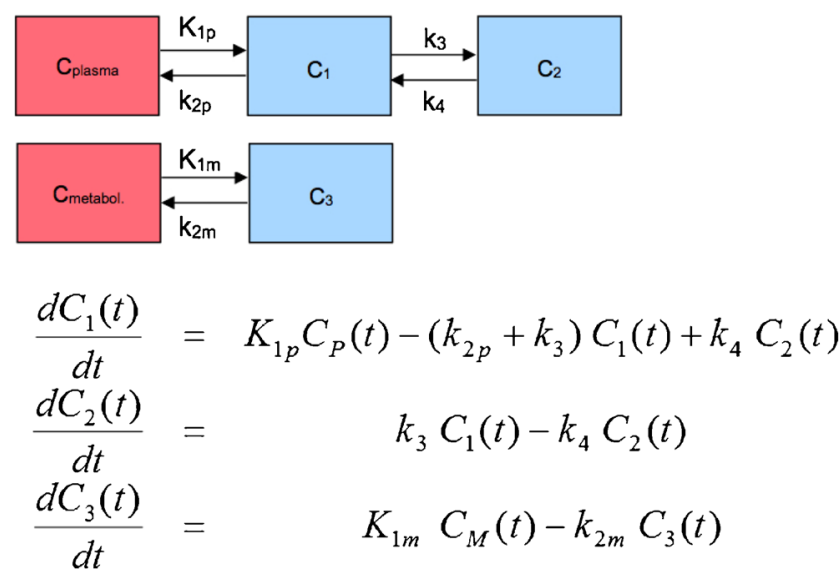

Fig. 4 Schematic presentation of a 3-compartment model with a double input function. If a labeled metabolite of the tracer enters tissue, the additional signal has to be accounted for in the model. This model includes a second input curve $C_{M}(t)$ of a metabolite entering tissue and undergoing nonspecific binding. $C_{1}$ represents the nondisplaceable compartment of the authentic ligand, $C_{2}$ the specific binding of interest, and $C_{3}$ metabolized ligand in tissue. $C_{P}(t)$ and $C_{M}(t)$ are the input curves of authentic ligand and metabolite, respectively. $C_{p} C_{\text {plasma }}, C_{M} C_{\text {metabolite }}$ 
activity was unmetabolized. The authors found a good correlation $\left(r^{2}=0.82\right)$ between the image-derived input function and the venous blood sampling, while the metabolic rate of the tracer correlated strongly with average SUV $\left(r^{2}=0.85\right)$ [39]. Other modifications have been proposed for a mathematical metabolite correction of receptor data by Burger and Buck using a series of ${ }^{11} \mathrm{C}$-iomazenil patient PET data [40]. Reference tissue compartmental methods have been proposed for the estimation of the binding potential from reversible ligand-receptor PET studies. In this case, instead of the use of an input VOI in a vessel, a reference region with no or very low specific uptake is used. Details about reference tissue compartmental modeling have been reported in the literature $[41,42]$. An overview of the different algorithms, which are in use for compartment modeling is provided elsewhere [43].

\section{Patlak-Gjedde plot}

The Patlak plot, also known as Patlak-Gjedde plot, is a graphical analysis technique based on a compartment model that uses linear regression to analyze the pharmacokinetics of a tracer. Prerequisite for the use of a Patlak plot is an irreversible trapping compartment of the tracer, as is assumed to be the case for ${ }^{18} \mathrm{~F}$-FDG [44]. The model is based on a blood/plasma compartment, a reversible and a nonreversible compartment, and can demonstrate whether the major metabolic step fits to a unidirectional transfer of the tracer, allowing the graphical calculation of an influx constant $\left(K_{i}\right)$. Importantly, since most biological reactions do not occur in a completely irreversible manner, Patlak and Blasberg had allready proposed in 1985 also a generalized version of this model, which could tolerate mild degrees of reversibility $\left(k_{4}>0\right)$ to avoid underestimation of the net uptake rate constant $K_{i}$ as previously reported for ${ }^{18} \mathrm{~F}$-FDG in certain regions, such as the normal liver, and tumor types, such as hepatocellular carcinoma [44]. This generalized version of the Patlak model was recently successfully applied for whole-body dPET and parametric imaging [45, 46].

The Patlak plot is calculated according to the formula:

$\frac{C_{T}(t)}{C_{P}(t)}=K \frac{\int_{0}^{t} C_{P}(\tau) d \tau}{C_{P}(t)}+V$

with $C_{P}(t)$ representing the input curve, $C_{T}(t)$ the measured tissue TAC, $K$ the slope, and $V$ the intercept.

The interpretation of $K$ and $V$ is based on the underlying compartment model. Particular in ${ }^{18}$ F-FDG, $K$ equals $K_{1} \times k_{3} /\left(k_{2}+k_{3}\right)$ and represents the metabolic flux of the tracer, while $V$ equals $V_{0}+V_{B}$, where $V_{0}$ represents the distribution volume of the reversible compartment $C_{1}$ and $V_{B}$ the fractional blood volume.
Moreover, the analysis of ${ }^{18} \mathrm{~F}$-FDG data requires the lumped constant (LC) and the plasma glucose level (PG) of the patient. The metabolic rate of glucose MRGlu is subsequently obtained from the regression slope by

$\mathrm{MRGlu}=K_{i} \frac{\mathrm{PG}}{\mathrm{LC}}$

\section{Noncompartmental models}

Fractal dimension Besides compartment analysis, a noncompartment model based on the fractal dimension (FD) can be applied. Fractal geometry is used to quantify structures that are poorly represented by the Euclidean geometry, for example for quantification of lesions with high structural complexity and irregular borders, and might therefore be helpful as an additional classification parameter [47].

FD is a parameter of heterogeneity and can also be used for temporal series, such as the time-activity data in each individual voxel of a VOI. The values of FD vary from 0 to 2 showing the deterministic or chaotic distribution of the tracer activity, with higher values reflecting more heterogeneous tracer distribution. Our group has evaluated TAC data derived from ${ }^{18} \mathrm{~F}$-FDG PET studies in 159 patients with 200 malignant lesions of different tumor entities as well as in 57 patients with 57 benign lesions for comparison. We used a box counting procedure as well as a subdivision of $7 \times 7$ and a maximal SUV of 20 for the calculation of FD, and found that FD demonstrated an accuracy of $77 \%$ for all patients, $68 \%$ for the untreated, and $83 \%$ for the treated group [48]. A FD cutoff value of 1.13 could reliably discriminate between malignant and benign lesions.

Fractal analysis has been furthermore used to quantify static whole-body images of patients with metastatic melanoma for immunotherapy monitoring after ipilimumab monotherapy [49]. In particular, the spreading of tumor cells was modeled via Monte Carlo simulations to address the evolution of the metastatic process and to predict the spatial distribution of metastatic lesions. Interestingly, FD was shown to decrease consistently with disease progression. Notably, problems existed in case of inflammatory lesions, like immune-related adverse events (e.g., colitis, thyroiditis), suggesting that areas with unspecific, nontumor-related uptake should be excluded for FD calculations. Overall however, the method is robust and operator independent and may be used as an additional tool for a multiparametric, PET-based, oncological assessment.

\section{Parametric imaging—-pixelwise modeling}

Parametric imaging is a method of feature extraction allowing the visualization of an isolated parameter of a tracer's kinetics based on dedicated mathematical models and a voxelwise 
calculation, instead of a VOI-based analysis. Parameters, which can be visualized, are the perfusion-related part of a tracer (transport), further metabolic steps - such as phosphorylation in the case of ${ }^{18}$ F-FDG-as well as the global influx. In case of receptor-specific tracers, parametric images of the receptor-binding or the internalization of the agent can be calculated. The advantage of parametric imaging as compared with the VOI-based image analysis is the calculation of images instead only of numbers. The most common approach is the usage of the reconstructed PET images and postprocessing of the data based on different algorithms. The first results of parametric imaging were published in 1992 by Messa et al. in patients with liver metastases [50].

Simplified parametric analysis of dPET data Parametric images can be calculated by fitting a linear regression function to the time-activity data on a pixel basis. Images of the slope and the intercept can then be calculated using dedicated software packages, such as the PMod software (PMOD Technologies Ltd., Zuerich, Switzerland) [51]. In particular, parametric images of the slope reflect primarily the transport/ phosphorylated part of ${ }^{18} \mathrm{~F}-\mathrm{FDG}$ and may be used for the delineation of suspicious tumor lesions due to the better contrast as compared with the SUV images (parametric images calculated from the original DICOM images by dividing them by injected dose normalized to body weight) or for the VOI placement in order to assess the trapped (phosphorylated) part of ${ }^{18} \mathrm{~F}$-FDG in a lesion. Respectively, parametric images of the intercept reflect the transport/perfusion-related part of ${ }^{18} \mathrm{~F}$ FDG, which is an indirect parameter of the perfusion of a lesion (Fig. 5). Some tumors, such as the giant cell tumors, are very clearly delineated in the intercept images of ${ }^{18} \mathrm{~F}$-FDG.
It was shown, that the enhanced perfusion related part of the

${ }^{18} \mathrm{~F}$-FDG in these tumors correlated to enhanced expression of genes related to angiogenesis, like the vascular endothelial growth factor A [52]. Futhermore, intercept images allow the visualization of vessels and can be used for the placement of input VOIs. Another advantage of this simplified parametric analysis of dPET data is that no input function is needed. Details of this method have been described elsewhere [53].

Patlak-based analysis Similar to compartmental modeling and in contrary to the simplified parametric analysis of dPET data, an input function is needed for the calculation of influx and intercept images according to Patlak analysis. Furthermore, the time frame used for the calculations should be defined by the operator. In general terms, it is recommended that the linear part of the TAC of a radiotracer should be used for Patlak analysis, when all reversible compartments are in equilibrium with plasma. In the case of ${ }^{18} \mathrm{~F}-\mathrm{FDG}$ this is approximately fulfilled during the last $30 \mathrm{~min}$ of the 60 -min dPET acquisition [44]. However, several investigators have used the dynamic data from the whole dPET acquisition for the calculation of parametric Patlak images, resulting in differences depending on the time frame used for the calculation. A dependence of the Patlak slope and intercept parameters and the post-injection time window of the dPET data from which the Patlak parameters were estimated, has been demonstrated. This time dependence of the supposedly time rate constant parameters of the Patlak model suggest that the conventional linear Patlak model may not always be the appropriate model for accurately describing the underlying tracer kinetics, for example due to the presence of nonnegligible uptake reversibility by the tracer under study $[45,54]$.
Fig. 5 Patient with a liver metastasis of rectal cancer following FOLFOX

chemotherapy. Fused transversal

${ }^{18} \mathrm{~F}$-FDG PET/CT image (left)

demonstrating enhanced uptake at the site of the metastasis $60 \mathrm{~min}$ p.i. Transversal SUV image 5060 min p.i. demonstrates an enhanced uptake (right upper row), while parametric image of the intercept (middle row) shows a decrease in the perfusion-related

${ }^{18} \mathrm{~F}$-FDG uptake, and parametric image of the slope (lower row) demonstrates an enhanced phosphorylation-related ${ }^{18} \mathrm{~F}-\mathrm{FDG}$ uptake

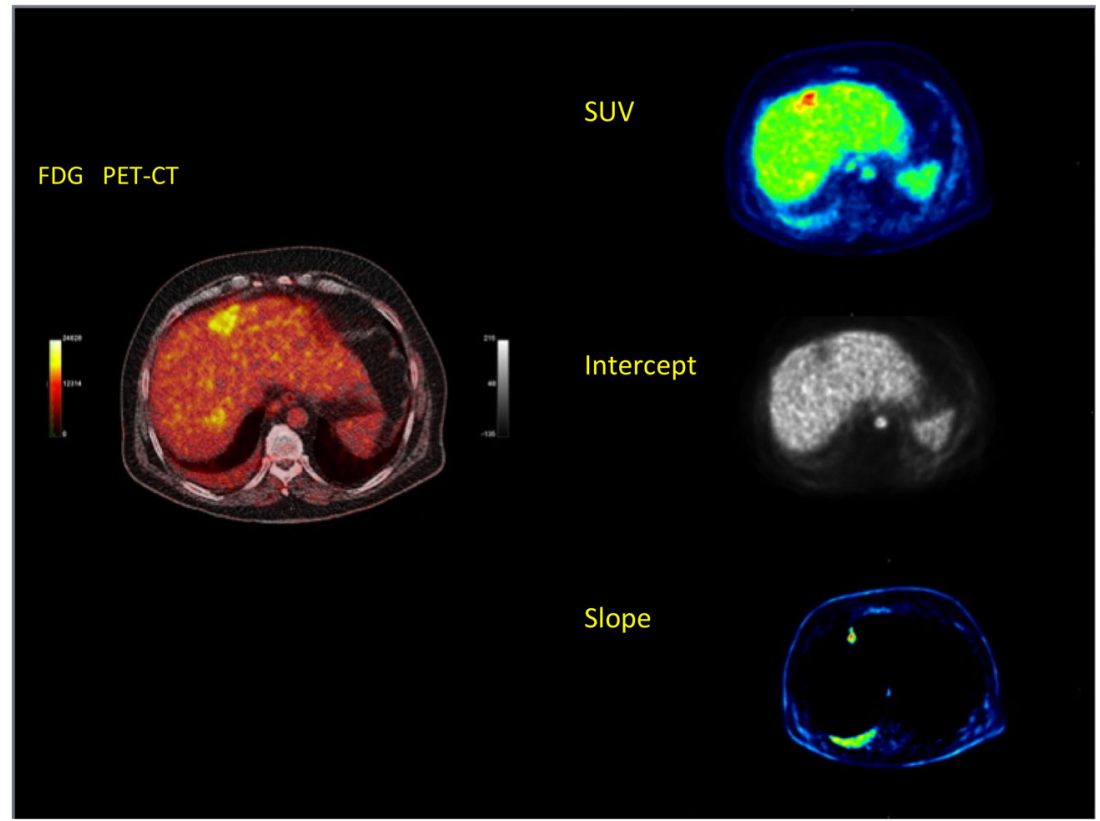


The intercept and influx images according to Patlak are related and very similar to the intercept and slope images based on the simplified parametric analysis model (Fig. 6).

Besides the standard linear Patlak graphical analysis, which does not take into account uptake reversibility, e.g., dephosphorylation in the case of ${ }^{18} \mathrm{~F}$-FDG, a nonlinear generalized Patlak method has been introduced by Patlak and Blasberg [44]. Moreover, Karakatsanis et al. proposed different Patlak-based parametric imaging methods for multibed dPET imaging. They compared the linear with the nonlinear graphical analysis and introduced a hybrid method consisting of a combination of linear and nonlinear Patlak analysis; this was achieved by applying nonlinear Patlak analysis selectively only to TACs from voxels exhibiting high Patlak correlation coefficients and linear Patlak to all other voxels. The authors recommend the use of nonlinear Patlak for highly quantitative imaging tasks, while for lesion detectability the hybrid technique is superior. Overall, all methods resulted in higher contrast-to-noise ratios as compared with SUV images [45].

In 2004, Zhu et al. developed a 4D linear Patlak algorithm for direct reconstruction from list-mode data across multiple bed positions based on a dual time point dynamic protocol instead of a full multiframe dynamic scan [55]. Recently, whole-body direct 4D parametric PET images employing nested generalized Patlak expectation-maximization reconstruction algorithms have been introduced and the first results demonstrate less noise in $\mathrm{K}_{\mathrm{i}}$ than the conventional postprocessing Patlak images [46]. The authors of this study propose a 7-frame protocol, consisting of an initial early dynamic scan (6 min) at the cardiac bed position immediately after the tracer injection for the calculation of the image-derived input function, followed by 6 whole-body dynamic scans over seven bed positions (40 min). However, the procedure needs a high computer capacity and a long reconstruction time. Some recently published studies have highlighted the clinical feasibility and impact of these approaches $[14,56]$.

Two- and three-tissue compartment model It is possible to calculate parametric images of the transport rates $K_{1}, k_{2}$, $k_{3}$, and $k_{4}$ as well as of the distribution volume $V_{B}$ based on a voxel-based application of the 3-tissue compartment described previously in this article. However, the robustness of the method is limited and needs further development. The advantage of the visualization of the transport rates and the distribution volume is the direct visual comparison of the rates in particular in follow-up studies, and theoretically the direct VOIbased calculation of the rates using the parametric images of each rate.

Fractal dimension Parametric images of FD can also be calculated [48]. As mentioned above, fractal geometry can be used to quantify lesions with high structural complexity and irregular borders, and might therefore be helpful as an additional parameter for the assessment of tracer heterogeneity in PET images. The example of Fig. 6 demonstrates a marked FD enhancement within a sarcoma of the left back muscles on the parametric image of FD, which is indicative for an enhanced heterogeneity in the tumor area (Fig. 6).

Principal component analysis Principal component analysis (PCA) is another noncompartment model, which describes the variance-covariance structure of a set of variables through some linear combinations of them. PCA has the main

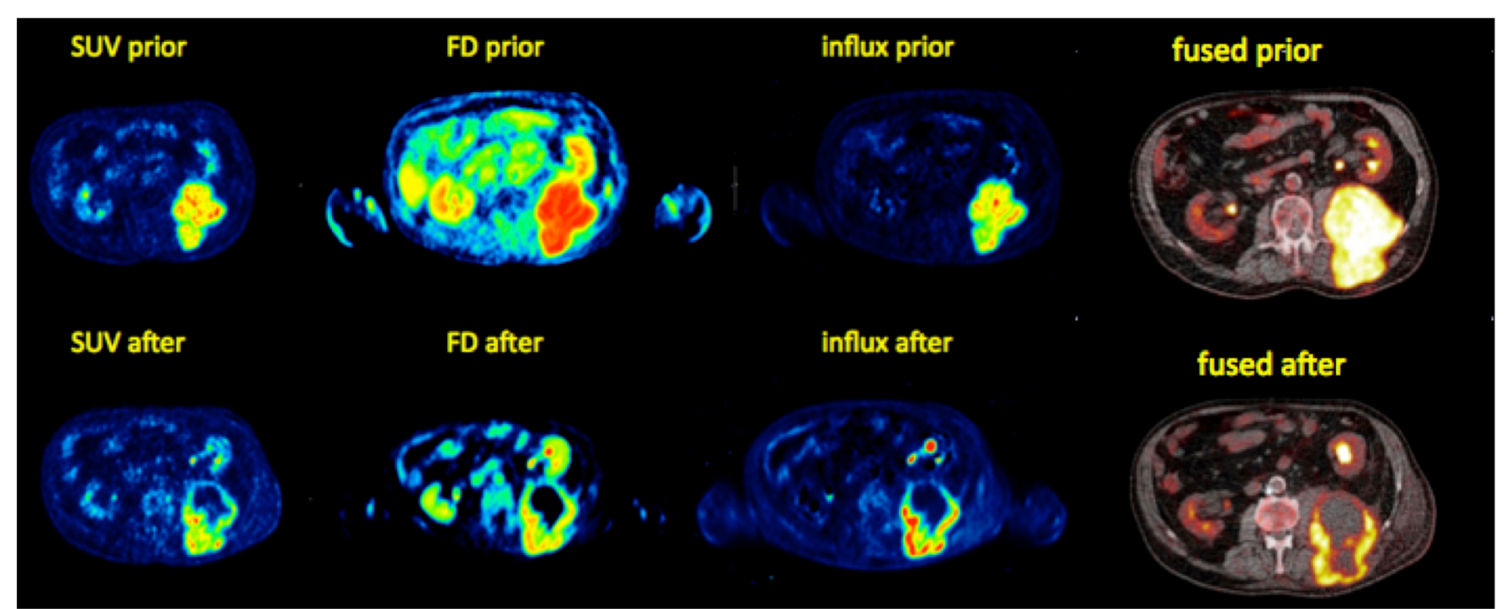

Fig. 6 Patient with an advanced high-grade retroperitoneal sarcoma infiltrating the left dorsal muscles. Tranversal ${ }^{18} \mathrm{~F}$-FDG PET images prior (upper row) and after the end of neoadjuvant pazopanib therapy (lower row) on the left part of the figure. The 55-60-min SUV images demonstrate a decrease of uptake and a central tumor necrosis as response to therapy (left). Comparable ${ }^{18} \mathrm{~F}$-FDG response pattern in the parametric images of the fractal dimension and the influx according to Patlak (middle). "Conventional," tranversal-fused PET/CT images on the right part 
objectives of data reduction and interpretation and can be used for parametric imaging. This method visualizes regions with different kinetics in a dynamic sequence by explaining the variance-covariance structure of the data set, leading to optimization of the signals by simultaneously considering the complete set of images in the dynamic sequence. Being independent of any kinetic model, PCA does not require the manual selection of VOIs and does not include any model-based restrictions. In general, only the first two to three components are useful for image interpretation: the first component is related to the perfusion in the case of ${ }^{18} \mathrm{~F}-\mathrm{FDG}$, whereas the second one is related to enhanced viability [57]. Methods for reduction of the background noise have been proposed to increase the image quality [58].

Similarity mapping Similarity mapping (SM) is a method of segmenting images into regions according to their temporal rather than spatial properties. Based on this approach, the similarity between the TAC of each pixel and the TAC of a reference ROI is calculated and displayed as an image. SM images provide spatially differentiated quantitative information describing the physiological behavior of the image structures, which sometimes may not be easily extracted from the visual inspection of dPET image sequences. Our group has reported on the application of this method in 20 patients with different malignancies who underwent ${ }^{18}$ F-FDG dPET [59]. In that study, SM supported the visual interpretation of PET data: based on the squared-sum normalized correlation coefficient, SM could identify structures with similar temporal properties to the tumor, enhancing the detection of metastases that were not easily depicted in the SUV images due to poor image quality or lesions' characteristics (size, location, etc.).

\section{Current clinical applications}

${ }^{18} \mathrm{~F}$-FDG PET/CT imaging has a great impact on the diagnostics and management of oncological patients and has gained tremendous use worldwide [60,61]. As mentioned above, most of the oncological studies are performed with static, whole-body PET/CT acquisitions, which assessment is mostly based on visual analysis and semi-quantitative evaluations by means of SUV calculations. In contrast, dPET imaging has been traditionally used for research purposes and is, thus, mainly performed in dedicated centers. A PubMed literature search with the keywords: compartment AND patients AND tumor AND PET revealed 204 papers (until October 2019). After exclusion of case reports, review articles and articles published in other languages than English 133 articles remained. The majority of these articles $(n=42)$ were based on dPET studies with ${ }^{18} \mathrm{~F}$-FDG and used 3-tissue compartment modeling, Patlak analysis, and fractal analysis. An overview of the radiotracers used, the tumors studies, the model used for kinetic analysis, and the goal of these studies is presented in Table 1.

The question raised by most physicians is when and why to use dPET imaging, given the fact that dynamic acquisition protocols are time consuming, the data processing and postprocessing are complicated, and it requires dedicated software tools beyond the ones regularly provided by the manufacturers. In the following paragraphs, the main applications of dPET - additional to the conventional, static PET imaging in several clinical settings are described.

\section{Diagnosis, staging, and tumor characterization}

One main clinical application of dPET is in the context of tumor diagnosis and staging. The concept behind this is the expansion of the diagnostic tools applied on the basis of a multiparametric image evaluation approach, including-besides SUV calculations-also kinetic data and parametric imaging. Several studies have highlighted the potential role of dPET imaging in initial tumor diagnosis and characterization. The vast majority of them were performed with the tracer ${ }^{18} \mathrm{~F}$-FDG.

Some of the first studies applying dPET in the oncological diagnostic workup were published by DimitrakopoulouStrauss et al. in patients with bone tumors and soft tissue sarcomas using ${ }^{18}$ F-FDG. The authors demonstrated that the combination of compartmental and SUV data leads to a higher discrimination between benign and malignant lesions, as well as to a more accurate tumor grading as compared with the use of SUV alone $[62,63]$. In bone tumors in particular, this multiparametric analysis was superior for the classification between grade I and grade III tumors with a positive predictive value $>80 \%$. The mean SUV, $V_{B}, K_{1}$, and $k_{3}$ were higher in malignant tumors compared with benign bone lesions. Overall, the combination of SUV, FD, $V_{B}, K_{1}, k_{2}, k_{3}$, and $k_{4}$ led to an accuracy of $87.7 \%$ in bone lesions as compared with $74.7 \%$ for SUV alone [62]. Regarding soft tissue tumors, $V_{B}$ and mean SUV were higher in sarcomas as compared with benign lesions. On the basis of six parameters of the ${ }^{18} \mathrm{~F}$ FDG kinetics (SUV, $V_{B}, K_{1}, k_{3}$, influx, FD), a better classification was achieved for soft tissue tumors with respect to grading as well as for differentiation between benign and malignant lesions. Interestingly, inflammatory lesions were misclassified, which was attributed to the similar ${ }^{18} \mathrm{~F}-\mathrm{FDG}$ kinetics between aggressive tumors and acute inflammations [63]. In another study of the same group, it was shown that patients with giant cell tumors demonstrated significantly enhanced $V_{B}$ as well as high $K_{1}$ and FD values as compared with other tumors such as soft-tissue sarcomas, which is of interest considering the classification of giant cell tumors as benign. This result was mainly attributable to an enhanced vascular fraction and increased ${ }^{18} \mathrm{~F}$-FDG transport of these tumors as was supported by gene chip data analysis, which revealed a close association between the kinetic ${ }^{18} \mathrm{~F}$-FDG data and the 


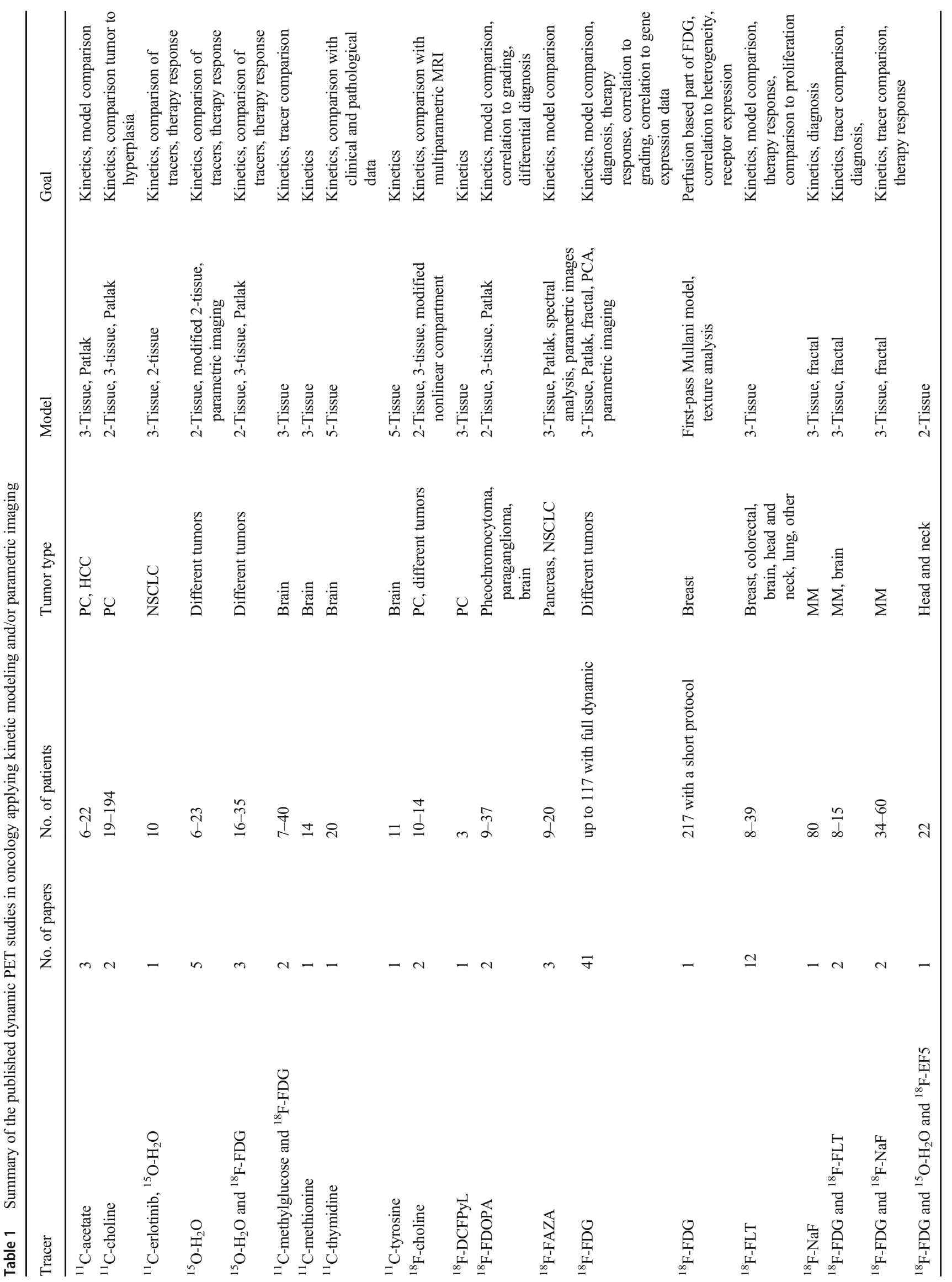




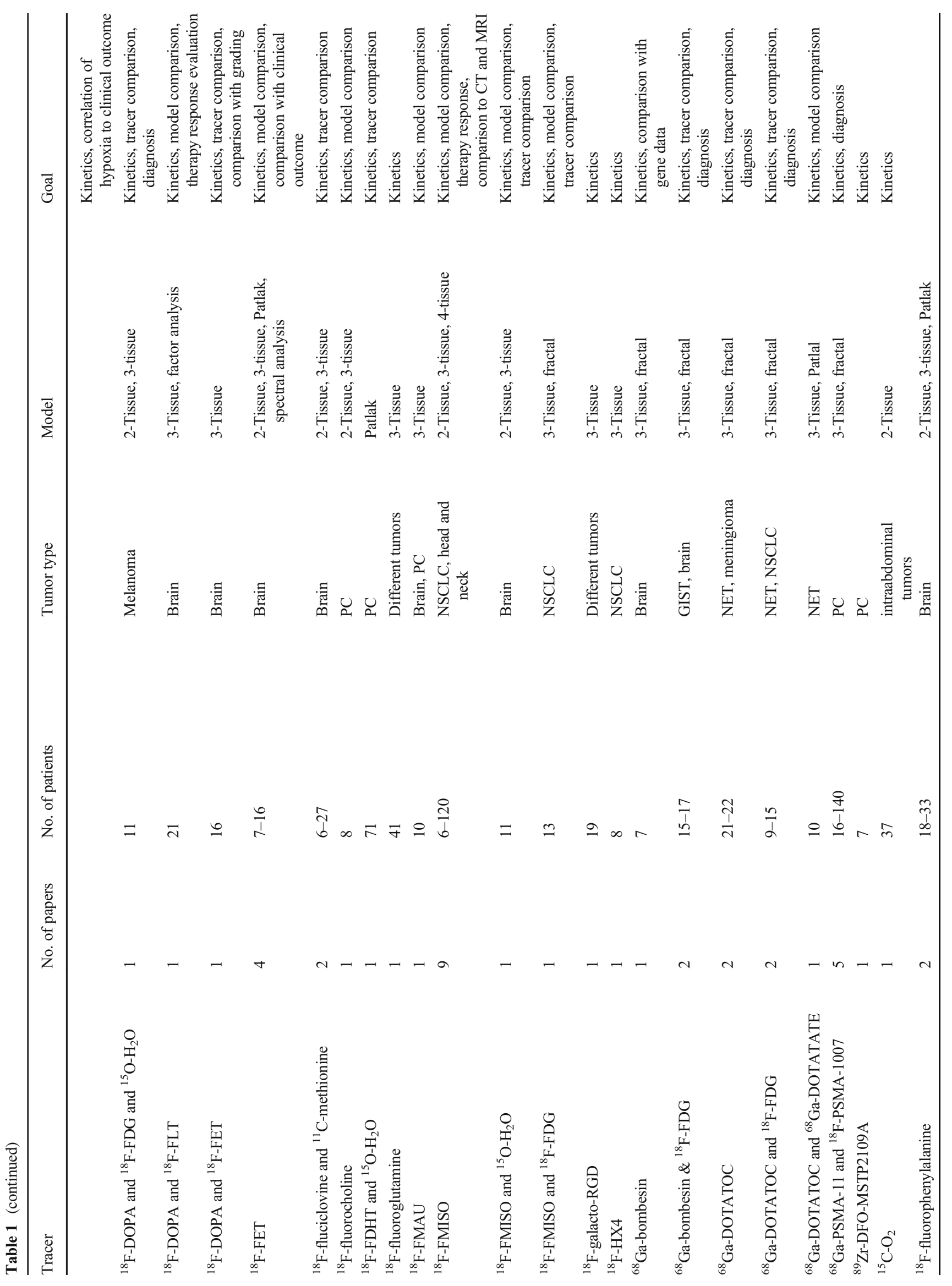




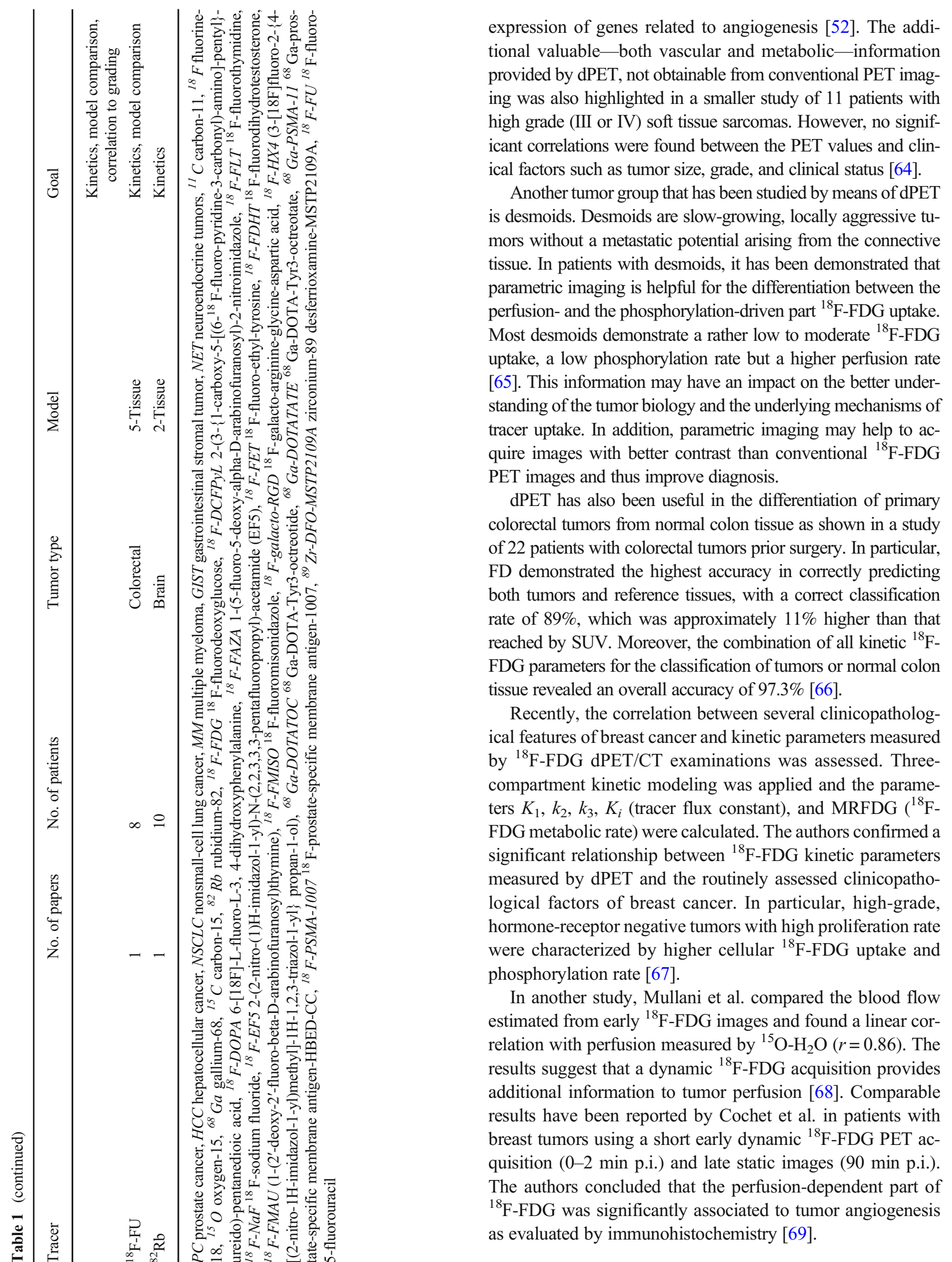


In the field of prostate cancer (PC), our group has recently studied with dynamic and static ${ }^{68} \mathrm{Ga}-\mathrm{PSMA}-11$ PET/CT a group of 16 patients with PC biochemical relapse attributed to local recurrence. Data analysis was performed by means of two-tissue compartment as well as parametric Patlak imaging. 12/16 patients were PSMA-positive in the static scans. Early dPET as well as parametric Patlak images detected an additional PC lesion not seen in static PET/CT due to its masking form urinary bladder activity. Based on these findings, it was assumed that early PET acquisitions and parametric Patlak images may have a potential for the detection of PC local recurrence [70].

\section{Therapy monitoring}

PET/CT with ${ }^{18} \mathrm{~F}-\mathrm{FDG}$ is an appropriate tool for therapy monitoring in a variety of tumors as well as in different therapeutic protocols including chemotherapy, radiotherapy and, most recently, immunotherapy $[60,71]$. In general, response assessment is based on visual evaluation of PET images and SUV calculations as well as on the application of known response evaluation criteria for PET, like the European Organization for Research and Treatment of Cancer for PET (EORTC) and the PET response criteria in solid tumors (PERCIST) $[72,73]$.

Response assessment based on kinetic data has been used for research purposes in limited numbers of patients. Our group performed dPET studies mainly with the radiotracer ${ }^{18} \mathrm{~F}-\mathrm{FDG}$ in patients with different tumors under several chemotherapeutic protocols. The most common observation of these studies was the overall increasing TACs prior to chemotherapy, which became decreasing or showed a plateau after treatment in responders [74, 75]. In particular, multiparametric analysis was applied in patients with metastatic colorectal cancer prior and within the course of FOLFOX chemotherapy; a combination of kinetic parameters derived from the baseline and a late follow-up ${ }^{18}$ F-FDG PET study (after 4 cycles) was better than the use of SUV alone for the classification of patients into a short or a long survival time (correct classification rate $78 \%$ vs. 69\%) [74]. The reason for this superiority of kinetic analysis vs. conventional SUV estimations may be that kinetic data can provide an early assessment of small metabolic changes, which cannot be detected by SUV. Moreover, ${ }^{18}$ F-FDG dPET has been shown helpful for the therapy assessment of patients with high-risk soft tissue sarcomas receiving neoadjuvant chemotherapy, as well as of those with metastatic soft tissue sarcomas being treated with high-dose chemotherapy and peripheral blood stem cell transplantation [75-77]. Particularly in chemotherapy, the combination of SUV and influx $\left(K_{i}\right)$ in the neoaduvant setting or SUV and $K_{1}$ in the adjuvant setting resulted in higher accuracy of response assessment than SUV alone (83\% vs. $67 \%$, and $90 \%$ vs. $85 \%$, respectively) $[75,76]$. In patients treated in a neoadjuvant setting with the tyrosine kinase inhibitor pazobanib, a significant decrease of the parameter $K_{1}$ was demonstrated before surgery, despite the lack of a statistically significant change in SUV. This decrease in $K_{1}$ was considered a potential marker in response to pazopanib due to the antiangiogenic effect of the therapeutic agent [77]. This finding is in accordance to dPET ${ }^{18} \mathrm{~F}$-FLT studies in patients with advanced solid tumors studied prior and after therapy with axitinib, a VEGFR-TK1 inhibitor. The assessment of tracer kinetics, based on 3-tissue compartment modeling, revealed a significant decrease in $V_{B}, K_{1}$, and $K_{i}$ as early as 2 weeks after therapy, as a sign of an anti-angiogenic and anti-proliferative effect [78].

Mankoff et al. performed dPET studies with ${ }^{15} \mathrm{O}-\mathrm{H}_{2} \mathrm{O}$ and ${ }^{18} \mathrm{~F}-\mathrm{FDG}$ in patients with locally advanced breast cancer prior and after neoadjuvant chemotherapy. They used a 2-tissue compartment for the evaluation of the perfusion studies and the metabolic rate based on Patlak graphical analysis, and reported a statistically significant trend for patients with a high metabolic rate to show a poorer response to chemotherapy. Furthermore, it was shown that a low ratio of the ${ }^{18} \mathrm{~F}-\mathrm{FDG}$ metabolic rate to blood flow was predictive of disease-free survival [79]. In another dPET study in breast cancer prior and after neoadjuvant chemotherapy, Humbert et al. assessed the perfusion-related part of ${ }^{18} \mathrm{~F}$-FDG using a first-pass model proposed by Mullani et al. after applying a short dynamic scan of 2 min starting with the tracer injection and a late scan 90 min p.i. [68]. Their data demonstrated a drastic reduction of the perfusion-related part of ${ }^{18}$ F-FDG only in HER-2 positive subtypes supporting the anti-angiogenic effect of trastuzumab. However, changes in SUVmax outperformed changes in perfusion effects for predicting pathological complete response in all tumor types [80].

Bahce et al. performed dPET studies with ${ }^{11} \mathrm{C}$-erlotinib and ${ }^{15} \mathrm{O}_{-} \mathrm{H}_{2} \mathrm{O}$ and applied compartmental analysis in 13 patients with advanced, epidermal growth factor receptor (EGFR)-mutated nonsmall-cell lung cancer [81]. Erlotinib is a tyrosine kinase inhibitor used for treatment of EGFR-mutated tumors. A subgroup of patients was scanned twice, prior, and 12 weeks after beginning of erlotinib. Although no significant change in the kinetic parameters of ${ }^{15} \mathrm{O}-\mathrm{H}_{2} \mathrm{O}$ was demonstrated, the distribution volume $\left(V_{B}\right)$ of ${ }^{11} \mathrm{C}$-erlotinib decreased as response to treatment. The authors suggested that this effect may be due to the occupancy of EGF receptors by the nonlabeled erlotinib, which was given together with the tracer.

In a study by Wardak et al., dynamic longitudinal studies with either ${ }^{18} \mathrm{~F}$-FLT or ${ }^{18} \mathrm{~F}$-fluoro-L-DOPA were performed in 21 patients with recurrent malignant glioma prior, after 2 , and 6 weeks after onset of treatment with bevacizumab (an angiogenesis inhibitor) and irinotecan (a chemotherapeutic agent). They showed that ${ }^{18} \mathrm{~F}$-FLT kinetic parameters early after onset of treatment were more predictive for overall survival than SUV. On the other hand, ${ }^{18}$ F-fluoro-L-DOPA information was inferior to ${ }^{18} \mathrm{~F}$-FLT [82]. 
In multiple myeloma (MM), a prospective study in 19 patients undergoing ${ }^{18} \mathrm{~F}$-FDG PET/CT before and after the first cycle of chemotherapy showed that changes in SUV and kinetics of the radiotracer could predict progression-free survival and identify patients who mostly benefited from therapy [83]. Furthermore, in a group of $34 \mathrm{MM}$ subjects undergoing high-dose chemotherapy and autologous stem-cell transplantation PET/CT studies with ${ }^{18} \mathrm{~F}$-FDG and the skeletal imaging tracer ${ }^{18} \mathrm{~F}-\mathrm{NaF}$ were performed before and after treatment. It was observed that SUV as well as kinetic parameters of ${ }^{18} \mathrm{~F}$ FDG and ${ }^{18} \mathrm{~F}-\mathrm{NaF}$ significantly decreased in all patients, who at the same time showed at least partial remission of the disease according to the clinical gold standard [84].

Another interesting field in terms of oncological therapy assessment is immunotherapy. The recent introduction and increasing application of immunotherapeutic agents in clinical practice has resulted in unprecedented improvements in patients' survival. Due to their unique mechanism of action, these novel agents have been associated with atypical response patterns by means of standard criteria. Thus, the application of conventional response criteria may misinterpret the effectiveness of immunotherapy. In an attempt to address this issue, dPET has also been employed. Nevertheless, the initial results were not satisfying. In particular, in patients suffering from stage IV metastatic melanoma being treated with ipilimumab immunotherapy, no superiority of dynamic ${ }^{18}$ F-FDG PET/CT as compared with static images could be demonstrated [85]. In this cohort of patients, the best criterion for immunotherapy response assessment was the number of new lesions detected on serial PET/CT imaging $[86,87]$. This fact may be melanoma-specific and related to the fact metastatic melanoma tends to metastasize rapidly in case of disease progression. Another recently proposed PET-based approach for prediction of eventual response in advanced melanoma patients under immunotherapy is the combination of anatomic (CT) and functional (PET) imaging parameters including SUV changes [88].

Overall, a multiparametric PET/CT evaluation based on a combination of SUV and kinetic data seems to be promising and superior to an assessment based merely on SUV calculations. Moreover, it is expected that the implementation of artificial intelligence in medical practice will facilitate and improve multiparametric approaches.

\section{Pharmacokinetic studies for tracer characterization}

Another main application of dPET is the pharmacokinetic characterization of new tracers. This can only be done by the use of dynamic scanning over a certain time depending on radiotracer studied. Most such studies have been performed either in preclinical models or in the field of neurosciences, for example, pharmacokinetic studies of dopamine, nicotinic acetylcholine or serotonin receptors as well as brain perfusion studies [89-92]. In the field of clinical oncology, besides ${ }^{18} \mathrm{~F}-\mathrm{FDG}$, more recently radiolabeled peptides have also been successfully studied with $\mathrm{dPET} / \mathrm{CT}$, such as somatostatin analogues (e.g., ${ }^{68} \mathrm{Ga}$-DOTATOC and ${ }^{68} \mathrm{Ga}$-DOTATATE) or PSMA radioligands (e.g., ${ }^{68} \mathrm{Ga}$-PSMA-11 and ${ }^{18}$ F-PSMA-1007). In the case of receptor-binding tracers, a two-tissue compartment model can be used as a simplification for the calculation of the rate constants and $V_{B}$. The interpretation of the rate constants is however different than that of ${ }^{18} \mathrm{~F}$-FDG, with $K_{1}$ reflecting the receptor binding, $k_{2}$ the displacement from the receptor, $k_{3}$ the cellular internalization, and $k_{4}$ its externalization.

Neuroendocrine tumors (NETs) of the gastrointestinal tract as well as meningiomas are ${ }^{68} \mathrm{Ga}$-DOTATOC avid. It has been demonstrated that untreated patients with these tumors show a continuous increase of ${ }^{68} \mathrm{Ga}$-DOTATOC $[17,93]$. Koukouraki et al. reported that $K_{1}$ had the greatest impact on the global ${ }^{68} \mathrm{Ga}$-DOTATOC SUV in NET followed by $V_{B}$ and $k_{3}$. Overall, pharmacokinetic analysis helped to separate blood background activity from receptor binding, which may have an impact for radionuclide therapy planning [17]. Ilan et al. compared the $K_{i}$ values based on kinetic data of a 3-tissue compartment model and the ones from parametric Patlak images for ${ }^{68} \mathrm{Ga}$-DOTATOC and ${ }^{68} \mathrm{Ga}$-DOTATATE in patients with metastatic NET and found a very high agreement [94]. In another study higher $K_{1} / k_{2}$ and $k_{3} / k_{4}$ values for ${ }^{68} \mathrm{Ga}$ DOTATOC in meningiomas as compared with reference tissue, as well as high $V_{B}$ values were reported, highlighting the more detailed analysis of the tumor biologic properties offered by pharmacokinetic modeling [93].

Sachpekidis et al. performed pharmacokinetic studies in PC patients with both ${ }^{68} \mathrm{Ga}$-PSMA-11 and ${ }^{18}$ F-PSMA-1007 [16, 95]. They reported on a continuous tracer increase up to $60 \mathrm{~min}$ as well as significantly higher kinetic values of ${ }^{68} \mathrm{Ga}$ PSMA-11 and ${ }^{18}$ F-PSMA-1007 in prostatic recurrence and metastatic lesions as compared with reference tissues. Comparable results have been reported by Schmuck et al. with ${ }^{68} \mathrm{Ga}$-PSMA11 and a short acquisition protocol (0-10-min dynamic acquisition and two late static images at 60 and $180 \mathrm{~min}$ p.i.) in 20 patients with primary PC. The authors concluded that early and delayed ${ }^{68} \mathrm{Ga}$-PSMA-11 images best discriminate $\mathrm{PC}$ within the prostatic gland [96]. Overall, the results are indicative for a high receptor binding and internalization of ${ }^{68} \mathrm{Ga}-\mathrm{PSMA}-11$ and ${ }^{18} \mathrm{~F}$ PSMA-1007 in prostate tumors and metastases, which may have potential applications in the field of PSMA radioligand therapy.

Pharmacokinetic results have been reported with the ${ }^{68} \mathrm{Ga}$ bombesin analog $\mathrm{BZH}_{3}$, which is a pan-bombesin analog that binds to at least three receptor subtypes, the neuromedin $\mathrm{B}$ (or $\mathrm{BB}_{1}$ ), the gastrin-releasing peptide or GRP (or $\mathrm{BB}_{2}$ ), and the bombesin receptor subtype $3\left(\mathrm{BB}_{3}\right)$. dPET studies with ${ }^{68} \mathrm{Ga}-$ $\mathrm{BZH}_{3}$ in patients with gastrointestinal stromal tumors (GISTs) demonstrated an enhanced accumulation in $41 \%$ of the patients and overall lower SUV and kinetic values for ${ }^{68} \mathrm{Ga}$ $\mathrm{BZH}_{3}$ as compared with ${ }^{18} \mathrm{~F}-\mathrm{FDG}$. The authors of that study concluded, that ${ }^{68} \mathrm{Ga}$-bombesin may be useful in a subgroup 
of GIST patients with low proliferation rate and therefore negative in ${ }^{18} \mathrm{~F}$-FDG PET [97]. In addition, in patients with recurrent gliomas ${ }^{68} \mathrm{Ga}-\mathrm{BZH} \mathrm{H}_{3}$ seems to be helpful for the differentiation between low- and high-grade gliomas based on a combination of kinetic ${ }^{18} \mathrm{~F}-\mathrm{FDG}$ and ${ }^{68} \mathrm{Ga}^{-} \mathrm{BZH}_{3}$ data. Overall, the ${ }^{68} \mathrm{Ga}_{-} \mathrm{BZH}_{3}$ accumulation was lower as compared with ${ }^{18}$ F-FDG [98].

A plethora of other pharmacokinetic studies with different radiotracers by means of dPET has been performed in patients with several malignancies. For example, ${ }^{18}$ F-fluroethyltyrosine (FET) and ${ }^{18}$ F-FLT have been used for the diagnosis of patients with gliomas [99, 100], while ${ }^{18}$ F-fluromisonidazole (MISO) has been successfully tested in different tumor entities like lung tumors and head and neck tumors for the determination of the hypoxic parts of the tumors [101-103]. Further, kinetic modeling has been used for the characterization of new tracers like

${ }^{18} \mathrm{~F}$-fluciclovine and ${ }^{18} \mathrm{~F}-\mathrm{HX} 4[104,105]$.

\section{Future perspectives}

dPET scanning is helpful for the diagnosis and therapy monitoring of oncological patients, but it is time-consuming and requires more complex evaluation techniques, which may hamper its routine use. Issues that are still open and need to be addressed include the following:

- The definition of shorter acquisition protocols including, e.g., a short dynamic acquisition immediately after tracer injection for the calculation of the input function and a short late dynamic acquisition 50-60 min p.i. We proposed a short dynamic acquisition $0-16 \mathrm{~min}$ and a late acquisition $60 \mathrm{~min}$ p.i. for ${ }^{18} \mathrm{~F}-\mathrm{FDG}$ and could demonstrate a high correlation between the kinetic data obtained from this short acquisition protocol as compared with a full dynamic series over $60 \mathrm{~min}$ [106]. Moreover as mentioned above, Karakatsanis et al. proposed a 7-frame protocol, consisting of an initial early dynamic scan (6 min) at the cardiac bed position followed by 6 whole-body dynamic scans over seven bed positions (40 $\mathrm{min}$ ) [46].

- The improvement of the evaluation software for dynamic images including sophisticated segmentation algorithms, automatic VOI placement, automatic calculation of TACs, implementation of validated methods for 2- and 3-tissue compartment modeling including a graphical interface for the users.

- Automatic calculation of the input function, either imagederived or population-based.

- Faster acquisition protocols and a potential implementation of whole-body parametric imaging, e.g., Patlak or simplified parametric model-based analysis for the new generation PET/CT scanners with an extended FOV.

- New reconstruction algorithms for the new generation $\mathrm{PET} / \mathrm{CT}$ scanners to improve counting statistics and image resolution. This issue can, however, be addressed with the introduction of clinically feasible dynamic whole-body PET imaging protocols in current generation limited axial FOV PET systems equipped with direct 4D reconstruction schemes and generalized nonlinear graphical analysis methods [8, 10, 107, 108].

- Implementation of artificial intelligence for the image analysis in order to facilitate and improve multiparametric approaches (combination of kinetic modeling and SUV measures).

The implementation of such quantitative approaches will need to be optimized, but it will open a new era for PET imaging. The adoption of such dynamic whole-body protocols, including parametric imaging, would facilitate the use of dPET initially in clinical studies for dedicated questions, and afterwards even into routine clinical protocols. Prerequisites for this wider usage of dPET include a further evidence of the added value and the gain in information offered by dPET compared with conventional static PET alone, and its acquisition in a patient- and operator-friendly manner.

\section{Limitations}

Dynamic PET/CT imaging for oncology including kinetic modeling and parametric imaging has been used until now primarily for research purposes and cannot be yet recommended for clinical use in its present form due to several limitations. Kinetic modeling needs further optimization to avoid overfitting, for example with the use of reference databases and SVM algorithms, as well as a robust definition of the input VOI. Furthermore, parametric images are noisy; thus, their interpretation should be done by experienced users and in comparison with conventional SUV images for reference. This limitation, however, is beginning to be addressed with the direct 4D parametric PET image reconstruction that has been introduced for single-bed and multibed dynamic PET studies, and which may allow the generation of parametric PET images of similar noise levels to those of conventional SUV images. In line with this, the industry has recently automated this technique to offer the automatic generation of direct Patlak parametric image and image-derived plasma input functions from dPET whole-body PET data. This effort represents a promising solution to facilitate the clinical translation of dPET imaging to clinical routine in oncology PET studies. Finally, another limitation is that although parametric imaging based on the Patlak approach and the simplified parametric analysis of dPET data have been thoroughly investigated in an experimental level, data are lacking on other algorithms like compartment modeling, FD, PCA, and SM. Overall, kinetic modeling and parametric imaging need further development and optimization of the algorithms used for calculation prior to their introduction in clinical practice. 


\section{Conclusion}

Multiparametric dPET based on kinetic modeling and parametric imaging of the applied radiotracers, offers a plethora of data not otherwise acquired with the conventional, static PET/ $\mathrm{CT}$. Its introduction in the diagnostic approach of the oncological patient is expected to provide superior information than the one derived from the visual evaluation of PET images merely supported by one semi-quantitative parameter, namely SUV. Although at present confined to research protocols, quantitative dPET and parametric imaging may gain importance and find increasing usage in the clinical routine as long as certain issues are addressed. This would be probably accomplished with the advent of the new generation PET/CT scanners and the expected improvement of the technical equipment, including an extended FOV, faster data acquisition and more sophisticated software for data evaluation.

Funding information Open Access funding provided by Projekt DEAL.

\section{Compliance with ethical standards}

Conflict of interest The authors declare that they have no conflict of interest.

Ethical approval This article is a review article and does not contain any studies with human participants or animals performed by any of the authors.

Open Access This article is licensed under a Creative Commons Attribution 4.0 International License, which permits use, sharing, adaptation, distribution and reproduction in any medium or format, as long as you give appropriate credit to the original author(s) and the source, provide a link to the Creative Commons licence, and indicate if changes were made. The images or other third party material in this article are included in the article's Creative Commons licence, unless indicated otherwise in a credit line to the material. If material is not included in the article's Creative Commons licence and your intended use is not permitted by statutory regulation or exceeds the permitted use, you will need to obtain permission directly from the copyright holder. To view a copy of this licence, visit http://creativecommons.org/licenses/by/4.0/.

\section{References}

1. Phelps ME, Hoffmann EJ, Na M, Ter-Pogossian MM. Application of annihilation coincidence detection to transaxial reconstruction tomography. J Nucl Med. 1975;16:210-24.

2. Sokoloff L, Reivich M, Kennedy C, et al. The (14C) deoxyglucose method for the measurement of local cerebral glucose utilization: theory, procedure and normal values in the conscious and anerstized albino rat. J Neurochem. 1977;28:897-916.

3. Phelps ME, Huang SC, Hoffman EJ, Selin C, Sokoloff L, Kuhl DE. Tomographic measurement of local cerebral glucose metabolic rate in humans with (F-18)2-fluoro-2-deoxy-glucose: validation of methos. Ann Neurol. 1979;6:371-88.

4. Wienhard K. measurements of glucose consumption using (18F)fluorodeoxyglucose. Methods. 2002;27:218-25.
5. Tomasi G, Turkheimer F, Aboagye E. importance of quantification for the analysis of PET data in oncology: review of current methods and trends for the future. Mol Imaging Biol. 2012;4:13146.

6. Badawi RD, Shi $\mathrm{H}, \mathrm{Hu} \mathrm{P}$, et al. First human imaging studies with the EXPLORER Total-body PET scanner. J Nucl Med. 2019;60: 299-303.

7. van Sluis J, Boellaard R, Dierckx RA, Stormezand G, Glaudemans AWJM, Noordzij W. Image quality and activity optimization in oncological 18F-FDG PET using the digital biograph vision PET/CT. J Nucl Med. 2019;jnumed.119.234351

8. Pantel AR, Viswanath V, Daube-Witherspoon ME, Dubroff JG, Muehllehner G, Parma MJ, et al. PennPET explorer: human imaging on a whole-body imager. J Nucl med. 2020;61(1):144-51.

9. Karp JS, Viswanath V, Geagan MJ, Muehllehner G, Pantel AR, Parma MJ, et al. PennPET explorer: design and preliminary performance of a whole-body imager. J Nucl med. 2020;61(1):13643.

10. Zhang X, Xie Z, Berg E, Judenhofer MS, Liu W, Xu T, et al. Total-body dynamic reconstruction and parametric imaging on the uEXPLORER. J Nucl med. 2020;61:285-91.

11. Karakatsanis, NA, Lodge MA, Zhou Y, Mhlanga J, Chaudhry MA, Tahari AK, et al. 2011, October. Dynamic multi-bed FDG PET imaging: feasibility and optimization. In 2011 IEEE nuclear science symposium conference record, pp. 3863-3870.

12. Karakatsanis NA, Lodge MA, Tahari AK, Zhou Y, Wahl RL, Rahmim A. Dynamic whole-body PET parametric imaging: I. concept, acquisition protocol optimization and clinical application. Phys Med Biol. 2013;58(20):7391.

13. Karakatsanis NA, Lodge MA, Zhou Y, Wahl RL, Rahmim A. Dynamic whole-body PET parametric imaging: II. Task-oriented statistical estimation. Phys Med Biol. 2013;58(20):7419.

14. Fahrni G, Karakatsanis NA, Di Domenicantonio G, Garibotto V, Zaidi H. Does whole-body Patlak 18 F-FDG PET imaging improve lesion detectability in clinical oncology? Eur Radiol. 2019;29(9):4812-21.

15. Rahmim A, Lodge MA, Karakatsanis NA, Panin VY, Zhou Y, McMillan A, et al. Dynamic whole-body PET imaging: principles, potentials and applications. Eur J Nucl Med Mol Imaging. 2019;46:501-18.

16. Sachpekidis C, Eder M, Kopka K, Mier W, Hadaschik BA, Haberkorn U, et al. (68)Ga-PSMA-11 dynamic PET/CT imaging in biochemical relapse of prostate cancer. Eur J Nucl Med Mol Imaging. 2016;43:1288-99.

17. Koukouraki S, Strauss LG, Georgoulias V, Eisenhut M, Haberkorn U. Dimitrakopoulou-Strauss a. comparison of the pharmacokinetics of $68 \mathrm{Ga}$-DOTATOC and (18F)FDG in patients with metastatic neuroendocrine tumours scheduled for $90 \mathrm{Y}$ DOTATOC therapy. Eur J Nucl Med Mol Imaging. 2006;33: 1115-20.

18. Graham MM, Lewellen BL. High-speed automated discrete blood sampling for positron emission tomography. J Nucl Med. 1993;34:1357-60.

19. Kanno I, Iida H, Miura S, Murakami M, Takahashi K, Sasaki H, et al. A system for cerebral blood flow measurement using an $\mathrm{H} 215 \mathrm{O}$ autoradiographic method and positron emission tomography. J Cerebr Blood Flow Metab. 1987;7:143-53.

20. van den Hoff J, Burchert W, Müller-Schauenburg W, Meyer GJ, Hundeshagen $\mathrm{H}$. accurate local blood flow measurements with dynamic PET: fast determination of input function delay and dispersion by multilinear minimization. J Nucl Med. 1993;34:17707.

21. Keiding S. bringing physiology into PET of the liver. J Nucl Med. 2012;53:425-33.

22. Turgeon V, Kertzscher G, Carroll L, Hopewell R, Massarweh G, Enger SA. Characterization of scintillating fibers for use as 
positron detector in positron emission tomography. Phys Med. 2019;65:114-20.

23. Ohtake T, Kosaka N, Watanabe T, Yokoyama I, Moritan T, Masuo M, et al. Noninvasive method to obtain input function for measuring glucose utilization of thoracic and abdominal organs. J Nucl Med. 1991;32:1432-8.

24. Miyazawa H, Osmont A, Petit-Taboue MC, Tillet I, Travere JM, Young AR, et al. Determination of 18F-fluoro-2-deoxy-D-glucose rate constants in the anesthetized baboon brain with dynamic positron tomography. J Neurosci Methods. 1993;50:263-72.

25. Sokoloff L, Smith CB. Basic principles underlying radioisotopic methods for assay of biochemical processes in vivo. In: Greitz T, Ingvar DH, Widén L, editors. The metabolism of the human brain studied with positron emission tomography. New York: Raven press; 1983. p. 123-48.

26. Feng ST, Cui M, Gao J, Wu B, Sha W, Huang B. Image-derived arterial input function in dynamic positron emission tomographycomputed tomography: a method using both positron emission tomographic and computed tomographic images. J Comput AssistTomogr. 2012;36:762-7.

27. Anazodo U, Kewin M, Finger E, Thiessen J, Hadway J, Butler L, et al. Preliminary evaluation of MRI-derived input function for quantitative measurement of glucose metabolism in an integrated PET-MRI. EJNMMI Phys. 2015;2:A80. https://doi.org/10.1186/ 2197-7364-2-s1-a80.

28. Naganawa M, Gallezot J-D, Shah V, et al. Assessment of population-based input functions for the Patlak plot using whole body 18F-FDG PET imaging. J Nucl Med. 2019;60(Suppl. 1): 519.

29. Rissanen E, Tuisku J, Luoto P, Arponen E, Johansson J, Oikonen $\mathrm{V}$, et al. Automated reference region extraction and populationbased input function for brain ((11C))TMSX PET image analyses. J Cereb Blood Flow Metab. 2015;35:157-65.

30. O'Sullivan F, Kirrane J, Muzi M, O'Sullivan JN, Spence AM, Mankoff DA, et al. Kinetic quantitation of cerebral PET-FDG studies withour concurrent blood sampling: statistical recovery of the arterial input function. IEEE Trans Med Imaging. 2010;29:610-24.

31. Reivich M, Alavi A, Wolf A, Fowler J, Russell J, Arnett C, et al. Glucose metabolic rate kinetic model parameter determination in humans: the lumped constants and rate constants for (18F)Fluorodeoxyglucose and (11C)Deoxyglucose. J Cereb Blood Flow Metab. 1985;5:179-92.

32. Hasselbalch SG, Madsen PL, Knudsen GM, Holm S, Paulson OB. Calculation of the FDG lumped constant by simultaneous measurements of global glucose and FDG metabolism in humans. J Cereb Blood Flow Metab. 1998;18:154-60.

33. Strauss LG, Koczan D, Klippel S, Pan L, Cheng C, Willis S, et al. Impact of angiogenesis-related gene expression on the tracer kinetics of 18F-FDG in colorectal tumors. J Nucl Med. 2008;49: $1238-44$.

34. Strauss LG, Koczan D, Klippel S, Pan L, Cheng C, Haberkorn U, et al. Impact of cell-proliferation-associated gene expression on 2deoxy-2-((18)f)fluoro-D-glucose (FDG) kinetics as measured by dynamic positron emission tomography (dPET) in colorectal tumors. Mol imaging biol. 2011;13:1290-300.

35. Burger C, Buck A. Requirements and implementation of a flexible kinetic modeling tool. J Nucl Med. 1997;38:1818-23.

36. Pan L, Mikolajczyk K, Strauss L, Haberkorn U. DimitrakopoulouStrauss a. machine learning based parameter imaging and kinetic modeling of PET data. J Nucl Med. 2007;48:158P.

37. Fujita M, Seibyl JP, Verhoeff NP, Ichise M, Baldwin RM, Zoghbi SS, et al. Kinetic and equilibrium analyses of [(123)I]epidepride binding to striatal and extrastriatal dopamine $\mathrm{D}(2)$ receptors. Synapse. 1999;34:290-304.
38. Muzi M, O’Sullivan F, Mankoff D, Doot R, Pierce L, Kurland B, et al. QIN: quantitative assessment of dynamic PET imaging data in cancer imaging. Magn Reson Imaging. 2013;30:1203-15.

39. Shields AF, Briston DA, Chandupatla S, Douglas KA, LawhornCrews J, Collins JM, et al. A simplified analysis of (18F)3-deoxy3 -fluorothymidine metabolism and retention. Eur J Nucl Med Mol Imaging. 2005;32:1269-75.

40. Burger C, Buck A. Tracer kinetic modellingo of receptor data with mathematical metabolite correction. Eur J Nucl Med. 1996;23: 539-45.

41. Lammertsma AA, Hume SP. Simplified reference tissue model for PET receptor studies. NeuroImage. 1996;4:153-8.

42. Cunningham VJ, Hume SP, Price GR, Ahler RG, Cremer JE, Jones AK. Compartmental analysis of diprenorphine binding to opiate receptors in the rat in vivo and its comparison with equilibrium data in vitro. J Cereb Blood Flow Metab. 1991;11:1-9.

43. Kotasidis FA, Tsoumpas C, Rahmin A. Advanced kinetic modelling strategies: towards adoption in clinical PET imaging. Clin Transl Imaging. 2014;2:219-37.

44. Patlak CS, Blasberg RG. Graphical evaluationof blood-to-brain transfer constants from multiple-time uptake data. Generalizations. J Cereb Flow Metab. 1985;5:584-90.

45. Karakatsanis NA, Zhou Y, Lodge MA, Casey ME, Wahl RL, Zaidi $\mathrm{H}$, et al. Generalized whole-body Patlak parametric imaging for enhanced quantification in clinical use. Phys Med Biol. 2015;60:8643-73.

46. Karakatsanis NA, Casey ME, Lodge MA, Rahmin A, Zaidi H. Whole-body direct 4D parametric PET imaging employing nested generalized Patlak expectation-maximization reconstruction. Phys Med Biol. 2016;61:5456-85.

47. Lennon FE, Cianci GC, Cipriani NA, Hensing TA, Zhang HJ, Chen C-T, et al. Lung cancer-a fractal viewpoint. Nat Rev Clin Oncol. 2015;12:664-75.

48. Dimitrakopoulou-Strauss A, Strauss LG, Burger C, Mikolajczyk $\mathrm{K}$, Lehnert T, Bernd $\mathrm{L}$, et al. On the fractal nature of positron emission tomography (PET) studies. World J Nucl Med. 2003;4: 306-13.

49. Breki CM, Dimitrakopoulou-Strauss A, Hassel J, Theoharis T, Sachpekidis C, Pan L, et al. Fractal and multifractal analysis of PET/CT images of metastatic melanoma before and after treatment with ipilimumab. EJNMMI Res. 2016;6:61.

50. Messa C, Choi Y, Hoh CK, Jacobs EL, Glaspy JA, Rege S, et al. Quantification of glucose utilization in liver metast ases: parametric imaging of FDG uptake with PET. J Comput Assist Tomogr. 1992;16:684-9.

51. Mikolajczyk K, Szabatin M, Rudnicki P, Grodzki M, Burger C. A Java environment for medical image data analysis: initial application for brain PET quantitation. Med Inform. 1998;23:207-14.

52. Strauss LG. Dimitrakopoulou-Strauss a, Koczan D, Bernd 1, Haberkorn U, Ewerbeck V et al. 18F-FDG kinetics and gene expression in giant cell tumors. J Nucl Med. 2004;45:1528-35.

53. Dimitrakopoulou-Strauss A, Hoffmann M, Bergner R, Uppenkamp M, Eisenhut M, Pan L, et al. Prediction of shortterm survival in patients with advanced nonsmall cell lung cancer following chemotherapy based on 2-deoxy-2-(F-18)-fluoro-Dglucose positron emission tomography: a feasibility study. Mol Imaging Biol. 2007;9:308-17.

54. Karakatsanis NA, Lodge MA, Casey ME, Zaidi H, Rahmim A. Impact of acquisition time-window on clinical whole-body PET parametric imaging. In 2014 IEEE nuclear science symposium and medical imaging conference (NSS/MIC), 2014;pp. 1-8.

55. Zhu W, Li Q, Bai B, Conti PS, Leahy RM. Patlak image estimation from dual time-point list-mode PET data. IEEE Trans Med Imaging. 2014;33:913-924.A.

56. Zhuang M, Karakatsanis NA, Dierckx RA, Zaidi H. Quantitative analysis of heterogeneous [18 F] FDG static (SUV) vs. Patlak (Ki) 
whole-body PET imaging using different segmentation methods: a simulation study. Mol Imaging Biol. 2019;21(2):317-27.

57. Thireou T, Strauss LG, Dimitrakopoulou-Strauss A, Kontaxakis G, Pavlopoulos S, Santos A. Performance evaluation of principal component analysis in dynamic FDG-PET studies of recurrent colorectal cancer. Comput Med Imaging Graph. 2003;27(1):4351.

58. Pedersen F, Bergström M. Bengtsson, Langström B. principal component analysis of dynamic positron emission tomography images. Eur J Nucl Med. 1994;21:1285-92.

59. Thireou T, Kontaxakis G, Strauss LG, Dimitrakopoulou-Strauss A, Pavlopoulos S, Santos A. Feasibility study of the use of similarity maps in the evaluation of oncological dynamic positron emission tomography images. Med Biol Eng Comput. 2005;43(1):23-32.

60. Dimitrakopoulou-Strauss A. PET-based molecular imaging in personalized oncology: potential of the assessment of therapeutic outcome. Future Oncol. 2015;5:127-42.

61. Dimitrakopoulou-Strauss A, Pan L, Strauss LG. Quantitative approaches of dynamic FDG-PET and PET/CT studies (dPET/CT) for the evaluation of oncological patients. Cancer Imaging. 2012;12:283-9.

62. Dimitrakopoulou-Strauss A, Strauss LG, Heichel T, Wu H, Burger C, Bernd L, et al. The role of quantitative (18)F-FDG PET studies for the differentiation of malignant and benign bone lesions. J Nucl Med. 2002;43:510-8.

63. Dimitrakopoulou-Strauss A, Strauss LG, Schwarzbach M, Burger C, Heichel T, Willeke F, et al. Dynamic PET 18F-FDG studies in patients with primary and recurrent soft-tissue sarcomas: impact on diagnosis and correlation with grading. J Nucl Med. 2001;42: 713-20.

64. Rusten E, Rødal J, Revheim ME, Skretting A, Bruland OS, Malinen E. Quantitative dynamic 18FDG-PET and tracer kinetic analysis of soft tissue sarcomas. Acta Oncol. 2013;52(6):1160-7.

65. Dimitrakopoulou-Strauss A, Hohenberger P, Pan L, Kasper B, Roumia S, Strauss LG. Dynamic PET with FDG in patients with unresectable aggressive fibromatosis: regression-based parametric images and correlation to the FDG kinetics based on a 2-tissue compartment model. Clin Nucl Med. 2012;37:943-8.

66. Strauss LG, Strauss LG, Klippel S, Pan L, Schönleben K, Haberkorn U, et al. Assessment of quantitative FDG PET data in primary colorectal tumours: which parameters are important with respect to tumour detection? Eur J Nucl Med Mol Imaging. 2007:34:868-77.

67. Kajáry K, Lengyel Z, Tökés AM, Kulka J, Dank M, Tökes T. Pathol Oncol Res. 2019. https://doi.org/10.1007/s12253-01900641-0.

68. Mullani NA, Herbst RS, O'Neil R, Gould KL, Barron BJ, Abbruzzese JL. Tumor blood flow measured by PET dynamic imaging of first-pass $18 \mathrm{~F}-\mathrm{FDG}$ uptake: a comparison with $15 \mathrm{O}$ labeled water-measured blood flow. J Nucl Med. 2008;49:51723.

69. Cochet A, Pigeonnat S, Khoury B, Vrigneaud JM, Touzery C, Berriolo-Riedinger A, et al. Evaluation of breast tumor blood flow with dynamic first-pass $18 \mathrm{~F}-\mathrm{FDG}$ PET/CT: comparison with angiogenesis markers and prognostic factors. J Nucl Med. 2012;53: 512-20.

70. Sachpekidis C, Pan L, Hadaschik BA, Kopka K, Haberkorn U, Dimitrakopoulou-Strauss A. 68Ga-PSMA-11 PET/CT in prostate cancer local recurrence: impact of early images and parametric analysis. Am J Nucl Med Mol Imaging. 2018;8:351-9.

71. Dimitrakopoulou-Strauss A. Monitoring of patients with metastatic melanoma treated with immune checkpoint inhibitors using PET-CT. Cancer Immunol Immunother. 2019;68:813-22.

72. Young H, Baum R, Cremerius U, Herholz K, Hoekstra O, Lammertsma AA, et al. Measurement of clinical and subclinical tumour response using (18F)-fluorodeoxyglucose and positron emission tomography: review and 1999 EORTC recommendations. European Organization for Research and Treatment of Cancer (EORTC) PET study group. Eur J Cancer. 1999;35: 1773-82.

73. Wahl RL, Jacene H, Kasamon Y, Lodge MA. From RECIST to PERCIST: evolving considerations for PET response criteria in solid tumors. J Nucl Med. 2009;50(Suppl 1):122S-50S.

74. Dimitrakopoulou-Strauss A, Strauss LG, Burger C, Rühl A, Irngartinger $\mathrm{G}$, Stremmel W, et al. Prognostic aspects of $18 \mathrm{~F}$ FDG PET kinetics in patients with metastatic colorectal carcinoma receiving FOLFOX chemotherapy. J Nucl Med. 2004;45:1480-7.

75. Dimitrakopoulou-Strauss A, Strauss LG, Egerer G, Vasamiliette J, Mechtersheimer G, Schmitt T, et al. Impact of dynamic 18F-FDG PET on the early prediction of therapy outcome in patients with high-risk soft-tissue sarcomas after neoadjuvant chemotherapy: a feasibility study. J Nucl Med. 2010;51:551-8.

76. Dimitrakopoulou-Strauss A, Strauss LG, Egerer G, Vasamiliette J, Schmitt T, Haberkorn U, et al. Prediction of chemotherapy outcome in patients with metastatic soft tissue sarcomas based on dynamic FDG PET (dPET) and a multiparameter analysis. Eur J Nucl Med Mol Imaging. 2010;37:1481-9.

77. Sachpekidis C, Karampinis I, Jakob J, Kasper B, Nowak K, Pilz L, et al. Neoadjuvant pazopanib treatment in high-risk soft tissue sarcoma: a quantitative dynamic 18F-FDG PET/CT study of the german interdisciplinary sarcoma group. Cancers. 2019;11:E790.

78. Scarpelli M, Simoncic U, Perlman S, Liu G, Jeraj R. Dynamic $18 \mathrm{~F}-\mathrm{FLT}$ imaging of spatiotemporal changes in tumor cell proliferation and vasculature reveals the mechanistic actions of antiangiogenic therapy. Phys Med Biol. 2018;63:155008.

79. Mankoff DA, Dunnwald LK, Gralow JR, Ellis GK, Charlop A, Lawton TJ, et al. Blood flow and metabolism in locally advanced breast cancer: relationship to response to therapy. J Nucl Med. 2002;43:500-9.

80. Humbert O, Lasserre M, Bertaut A, Fumoleau P, Coutant A, Brunotte $\mathrm{F}$, et al. Breast cancer blood flow and metabolism on dual-acquisition 18F-FDG PET: correlation with tumor phenotype and neoadjuvant chemotherapy response. J Nucl Med. 2018;59: 1035-41.

81. Bahce I, Yaqub M, Errami H, Schuit RC, Schober P, Thunnissen E, et al. Effects of erlotinib therapy on (11C)erlotinib uptake in EGFR mutated, advanced NSCLC. EJNMMI Res. 2016;6:10.

82. Wardak M, Schiepers C, Cloughesy TF, Dahlbom M, Phelps ME, Huang SC. 18F-FLT and 18F-FDOPA PET kinetics in recurrent braiun tumors. Eur J Nucl Med Mol Imaging. 2014;41:1199-209.

83. Dimitrakopoulou-Strauss A, Hoffmann M, Bergner R, Uppenkamp M, Haberkorn U, Strauss LG. Prediction of progression-free survival in patients with multiple myeloma following anthracycline-based chemotherapy based on dynamic FDG-PET. Clin Nucl Med. 2009;34(9):576-84.

84. Sachpekidis C, Hillengass J, Goldschmidt H, Wagner B, Haberkorn U, Kopka K, et al. Treatment response evaluation with $18 \mathrm{~F}-\mathrm{FDG} \mathrm{PET} / \mathrm{CT}$ and $18 \mathrm{~F}-\mathrm{NaF} \mathrm{PET} / \mathrm{CT}$ in multiple myelomapatients undergoing high-dose chemotherapy and autologous stem cell transplantation. Eur J Nucl med Mol imaging. 2017;44(1):50-62.

85. Sachpekidis C, Anwar H, Winkler JK, Kopp-Schneider A, Larribere L, Haberkorn U, et al. Longitudinal studies of the $18 \mathrm{~F}$ FDG kinetics after ipilimumab treatment in metastatic melanoma patients based on dynamic FDG PET/CT. Cancer Immunol Immunother. 2018;67:1261-70.

86. Anwar H, Sachpekidis C, Winkler J, Kopp-Schneider A, Haberkorn U, Hassel JC, et al. Absolute number of new lesions on $18 \mathrm{~F}-\mathrm{FDG} \mathrm{PET} / \mathrm{CT}$ is more predictive of clinical response than SUV changes in metastatic melanoma patients receiving ipilimumab. Eur J Nucl Med Mol Imaging. 2018;45:376-83. 
87. Sachpekidis C, Anwar H, Winkler J, Kopp-Schneider A, Larribere $\mathrm{L}$, Haberkorn U, et al. The role of interim 18F-FDG PET/CT in prediction of response to ipilimumab treatment in metastatic melanoma. Eur J Nucl Med Mol Imaging. 2018 Jul;45(8):1289-96.

88. Cho SY, Lipson EJ, Im HJ, Rowe SP, Gonzalez EM. Blackford a, et al. Prediction of response to immune checkpoint inhibitor therapy using early-time-point 18F-FDG PET/CT imaging in patients with advanced melanoma. J Nucl Med. 2017 Sep;58(9):1421-8.

89. Lammertsma AA, Hume SP. Simplified reference tissue mode for PET receptor studies. Neuroimage. 1996;4:153-8.

90. Hillmer AT, Esterlis I, Gallezot JD. bois F, Zheng MQ, Nabulsi N, et al. Imaging of cerebral $\alpha 4 \beta 2 *$ nicotinic acetylcholine receptors with $(-)-(18$ F)Flubatine PET: implementation of bolus plus constant infusion and sensitivity to acetylcholine in human brain. Neuroimage. 2016;141:71-80.

91. Ichise M, Liow JS, Lu JQ, Takano A, Model K, Toyama H, et al. Linearized reference tissue parametric imaging methods: application to (11C)DASB positron emission tomography studies of the serotonin transporter in human brain. J Cereb Blood Flow Metab. 2003;23:1096-112.

92. Raichle ME, Martin WRW, Herscovitch P, Mintun MA, Markham J. Brain blood flow measured with intravenous $\mathrm{H} 2 \mathrm{O} 15$. II. Implementation and validation. J Nucl Med. 1983;24:790-8.

93. Henze M, Dimitrakopoulou-Strauss A, Milker-Zabel S, Schuhmacher J, Strauss LG, Doll J, et al. Characterization of 68Ga-DOTA-D-Phe1-Tyr3-octeotride kinetics in patients with meningiomas. J Nucl Med. 2005;46:763-9.

94. Ilan E, Sandström M, Velikyan I, Sundin A, Eriksson B, Lubberink M. Parametric net imflux rate images of $68 \mathrm{Ga}-$ DOTATOC and 68Ga-DOTATATE: quantitative accuracy and improved image contrast. J Nucl Med. 2017;58:744-9.

95. Sachpekidis C, Afshar-Oromieh A, Kopka K, Strauss DS, Pan L, Haberkorn U, et al. 18F-PSMA-1007 multiparametric, dynamic $\mathrm{PET} / \mathrm{CT}$ in biochemical relapse and progression of prostate cancer. Eur J Nucl Med Mol Imaging. 2019. https://doi.org/10.1007/ s00259-019-04569-0.

96. Schmuck S, Mamach M, Wilke F, von Klot CA, Henkenberens C, Thackeray JT, et al. Multiple time-point 68Ga-PSMA I\&T PET/ CT for characterization of primary prostate Cancer: value of early dynamic and delayed imaging. Clin Nucl Med. 2017;42:286-93.

97. Dimitrakopoulou-Strauss A, Hohenberger P, Haberkorn U, Mäcke HR, Eisenhut M, Strauss LG. 68Ga-labeled Bombesin studies in patients with gastrointestinal stromal tumors: comparison with 18F-FDG. J Nucl Med. 2007;48:1245-50.

98. Dimitrakopoulou-Strauss A, Seiz M, Tuettenberg J, Schmieder K, Eisenhut M, Haberkorn U, et al. Pharmacokinetic studies of 68Ga- labeled Bombesin (68Ga-BZH3) and F-18 FDG PET in patients with recurrent gliomas and comparison to grading. Clin Nucl Med. 2011;36:101-8.

99. Koopman T, Verburg N, Pouwels PJ, Wesseling P, Hoekstra OS, De Witt Hamer PC, et al. Quantitative parametric maps of O-(2(18F)fluoroethyl)-L-tyrosine kinetics in diffuse gliomas. J Cereb Blood Flow Metab. 2019; may 24:271678X19851878

100. Kudomi N, Maeda Y, Hatakeyama T, Yamamoto Y, Nishiyama Y. Fully parametric imaging with reversible tracer $18 \mathrm{~F}-$ FLT within a reasonable time. Radiol Phys Technol. 2017;10:41-8.

101. Sachpekidis C, Thieke C, Askoxylakis V, Nicolay NH, Huber PE, Thomas M, et al. Combined use of (18)F-FDG and (18)F-FMISO in unresectable non-small cell lung cancer patients planned for radiotherapy: a dynamic PET/CT study. Am J Nucl Med Mol Imaging. 2015;5(2):127-42.

102. Schwartz J, Grkovski M, Rimner A, Schroeder H, Zanzonico PB, Carlin SD, et al. Pharmacokinetic analysis of dynamic 18FFluoromisonidazole PET data in non-small cell lung cancer. J Nucl Med. 2017;58:911-9.

103. Grkovski M, Lee NY, Schroeder H, Carlin SD, Beattie BJ, Riaz N, et al. Monitoring early response to chemoradiotherapy with $18 \mathrm{~F}$ MISO dynamic PET in head and neck cancer. Eur J Nucl Med Mol Imaging. 2017;44:1682-91.

104. Michaud L, Beattie BJ, Akhurst T, Dunphy M, Zanzonico P, Finn $\mathrm{R}$, et al. 18F-Fluciclovine (18F-FACBC) PET imaging of recurrent brain tumors. Eur J Nucl Med Mol Imaging. 2019. https://doi. org/10.1007/s00259-019-04433-1.

105. Verwer EE, Zegers CM, van Elmpt W, Wierts R, Windhorst AD, Mottaghy FM, et al. Pharmacokinetic modeling of a novel hypoxia PET tracer (18F)HX4 in patients with non-small cell lung cancer. EJNMMI Phys. 2016;3(1):30.

106. Strauss LG, Pan L, Cheng C, Haberkorn U. DimitrakopoulouStrauss A. Shortened acquisition protocols for the quantitative assessment of the 2-tissue-compartment model using dynamic PET/CT 18F-FDG studies. J Nucl Med. 2011;52:379-85.

107. Van Sluis J, de Jong J, Schaar J, Noordzij W, van Snick P, Dierckx $\mathrm{R}$, et al. Performance characteristics of the digital biograph vision PET/CT system. J Nucl Med. 2019;60:1031-6.

108. Zein SA, Karakatsanis NA, Issa M, Haj-Ali AA, Nehmeh SA. Physical performance of a long axial field of view PET scanner prototype with sparse rings configuration: a Monte Carlo simulation study. Med Phys. 2020. https://doi.org/10.1002/mp.14046.

Publisher's note Springer Nature remains neutral with regard to jurisdictional claims in published maps and institutional affiliations. 